\title{
4. Eugenik und die Frage nach Anlage oder Umwelt (1860-1930)
}

\subsection{Gentleman scientist: Francis Galton und das Geheimnis der Vererbung}

Die Ursprünge der englischen Eugenik sind eng mit dem Namen Francis Galton verbunden. ${ }^{1}$ Den hochbegabten Cousin von Charles Darwin zeichnete eine universale wissenschaftliche Neugierde ${ }^{2}$ und trat mit der Entwicklung einer ganzen Reihe von Untersuchungsmethoden zum Vererbungsmechanismus und der Identifizierung von Kriminellen in Erscheinung, die in den wissenschaftlichen und populärwissenschaftlichen Kriminalitätsdebatten der Jahrhundertwende eine bedeutende Rolle spielten. Ein Blick auf seinen Werdegang und die Entwicklung seiner Theorien zur Vererbung soll helfen, die Grundlagen dieser Debatten besser verstehen zu können. Ähnlich wie Darwin verkörperte Galton den Typus des gentleman scientist, der sich, finanziell durch privates Vermögen abgesichert, ungehindert seinen wissenschaftlichen Interessen widmen konnte. Galtons $\mathrm{Zu}$ sammenarbeit mit zahlreichen wissenschaftlichen Institutionen und staatlichen Einrichtungen demonstriert darüber hinaus das für Großbritannien lange Zeit typische Beispiel einer erfolgreichen Kooperation zwischen einem institutionell nicht gebundenen Wissenschaftler und privaten und staatlichen Foren der Vermittlung und Popularisierung von Wissen. ${ }^{3}$ Die Aneignung und Instrumentalisierung der von Galton entwickelten Eugenik durch eine von ihm unabhängige politische Pressuregroup, die 1907 gegründete Eugenics Education Society, ist ein weiteres Beispiel dafür, auf welche Weise singulär produziertes Wissen breitenwirksam rezipiert werden konnte. Anhand der Eugenics Education Society lassen

1 Das Leben und Wirken Francis Galtons ist bereits Gegenstand intensiver Forschung geworden; zu den jüngeren biographischen Arbeiten gehören: DereK William ForReST, Francis Galton: The Life and Work of a Victorian Genius, London und New York 1974; NichOLAS Wright Gillham, A Life of Sir Francis Galton: From African Explorer to the Birth of Eugenics, Oxford 2001; Michael Bulmer, Francis Galton. Pioneer of Heredity and Biometry, Baltimore und London 2003; MARTIN BRoOKES, Extreme Measure. The dark Visions and bright Ideas of Francis Galton, London 2004 erhebt keinen wissenschaftlichen Anspruch. Eine Fundgrube (auch in Bezug auf Abbildungen) sind dagegen die von Galtons Schüler Karl Pearson herausgegebenen Bände: KARL PEARSON, The Life, Letters and Labours of Francis Galton, 1. Bd.: Birth 1822 to Marriage 1853; 2. Bd.: Researches in Middle Life; 3. Bd.: (a) Correlation, Personal Identification and Eugenics, (b) Characterisation, especially by letters, Cambridge 1914/1924/1930.

2 So der Titel eines Aufsatzes von Milo Keynes, Sir Francis Galton - A Man with a Universal Scientific Curiosity, in: Milo Keynes (Hrsg.), Sir Francis Galton, FSR: The Legacy of His Ideas, London 1993, S. 1-32.

3 Vgl. dazu Sabine Freitag und MoniKa Löscher, Vereine als Vermittler zwischen Wissenschaft und Öffentlichkeit, in: Sybilla Nikolow und Arne Schirrmacher (Hrsg.), Wissenschaft und Öffentlichkeit als Ressourcen füreinander. Studien zur Wissenschaftsgeschichte im 20. Jahrhundert, Frankfurt am Main und New York 2007, S.339-346. 
sich insbesondere die Grenzen der Verankerung biologistischer Argumente in englischen Kriminalitätsdiskursen aufzeigen.

In seinen Memoiren hat Francis Galton mehrfach darauf hingewiesen, wie wichtig die Publikation von Darwins On the Origin of Species im Jahre 1859 für die Entwicklung seiner eigenen wissenschaftlichen Laufbahn gewesen sei: „[It] made a marked epoch in my own mental development, as it did in human thought generally. " 4 Bis zu diesem Zeitpunkt hatte Galton seine zweifellos zahlreichen Talente auf verschiedenen Forschungsgebieten erprobt und sich unter anderem mit Geologie, Kartographie und Meteorologie beschäftigt. ${ }^{5}$ Eine vierjährige Forschungsreise durch das tropische südliche Afrika hatte ihn zu diesen Themen angeregt. ${ }^{6}$ Warum sich Galton plötzlich von diesen Forschungsgebieten abwandte, um sich ganz auf das Thema seines Lebens - Vererbung - zu konzentrieren, ist in der Literatur umstritten. Persönliche und intellektuelle Motive dürften dabei eine gleichermaßen große Rolle gespielt haben. ${ }^{7}$ Ohne Frage aber war es besonders seine Darwin Lektüre, die Galton darin bestärkte, „Heredity and the possible improvement of the Human Race" ${ }^{\text {"8 }}$ zu seinem zentralen Forschungsschwerpunkt auszubauen.

Wie viele seiner Zeitgenossen hatte auch Galton die tief greifenden und beunruhigenden Veränderungen eines beschleunigten Industriezeitalters mit Sorge zur Kenntnis genommen. Ähnlich wie viele Statistiker und Gefängnisärzte war er der Auffassung, dass im modernen urbanen Leben nach den Ursachen für die degenerierenden Effekte gesucht werden müsse, die sich vor allem in den unteren

4 Francis Galton, Memories of my Life, London ${ }^{3} 1909$, S. 287 f., hier zit. nach Gillham, A Life of Sir Francis Galton, S. 155.

5 In ihren Erinnerungen schrieb Beatrice Webb über Francis Galtons (1822-1911): „[T]he most relevant of Galton's many gifts was the unique contribution of three separate and distinct processes of the intellect; a continious curiosity about, and rapid apprehension of individual facts, whether common or uncommon; the faculty for ingenious trains of reasoning; and; more admirable than either of these [...] the capacity for correcting and verifying his own hypotheses, by the statistical handling of masses of data, whether collected by himself or supplied by other students of the problem." BEATrice WebB, My Apprenticeship, Bd. 1, Harmondsworth 1938, S. 158; zu Galtons Talenten auch LESLIE S. HEARNSHAW, A Short History of British Psychology, 1840-1940, London 1964, S. 56-66: „For Galton was unquestionable a genius and, [...] his genius manifested itself in most varied fields" (ebd., S. 56).

$6 \mathrm{Zu}$ Galtons Afrikareisen siehe Gillham, Francis Galton, Kap. 2: Geography and Exploration, S. 61-154; auch ForRest, Francis Galton.

7 Neil Davie äußert die Vermutung, dass Galtons Interesse an Vererbung mit der Kinderlosigkeit seiner eigenen Ehe zusammenhing, wobei er auch darauf aufmerksam macht, dass alle Brüder Galtons und alle Schwestern seiner Frau ebenfalls kinderlos blieben (vgl. Davie, Tracing the Criminal, S. 243). Daniel Kelves hat darauf hingewiesen, dass Galton sich vorzugsweise Forschungsgebiete ausgesucht habe, die noch relativ ,unbesetzt ' gewesen seien, Galton also wenig Konkurrenz, Widerspruch und Kritik habe erwarten müssen (DANIEL KeLVES, In the Name of Eugenics, New Haven ${ }^{2} 1995$, S. 8-11); Interesse an psychischen Vorgängen und Fehlleistungen gehen nach Meinung von Nicholas Gillham auch auf Galtons eigenen Nervenzusammenbruch während seiner Studienzeit in Cambridge zurück, der ihn zwang, sein Studium zu unterbrechen, um auf Reisen Erholung zu suchen. Zugleich habe dieser Zusammenbruch aber intellektuelle Selbstzweifel hinterlassen (vgl. Gillham, Francis Galton, S.45, S. 150f.).

8 Galton, Memories of my Life, S. 288. 
Schichten beobachten ließen: „It is perfectly distressing to me“, so schrieb er 1869, "to witness the draggled, drudged, mean look of the mass of individuals, especially of women, that one meets in the streets of London and other purely English towns. The conditions of their life seem too hard for their constitutions, and to be crushing them into degeneracy." ${ }^{\text {"9 }}$ Doch während der britische Sozialphilosoph Herbert Spencer in einer Kombination aus Darwin und Degeneration den Begriff des survival of the fittest prägte und darauf setzte, dass im natürlichen Selektionsprozess ohnehin nur die Angepasstesten und Fähigsten überleben und die Schwachen sterben würden, aktive Eingriffe also überflüssig seien, ${ }^{10}$ und Morel das Problem relativierte, indem er die vierte Generation von Degenerierten ohnehin für steril hielt, wollte Francis Galton den Dingen nicht einfach ihren Lauf lassen, standen doch Wettbewerbsfähigkeit und Fortschritt Großbritanniens auf dem Spiel. ${ }^{11}$ Sehr früh schon, in den 1860er Jahren, formulierte er Ideen zu kontrollierten und gezielten Reproduktionsprogrammen, die sicherstellen sollten, dass ,Charakter ${ }^{`}$ und ,Talent' sich durchsetzen konnten. ${ }^{12}$

Wissenschaftlich interessierte sich Galton von Anfang an mehr für die Vererbung intellektueller Fähigkeiten und Kapazitäten als für die Vererbung äußerlicher Merkmale. Doch wie ließ sich beweisen, dass intellektuelle Fähigkeiten erblich bedingt waren? Galton besann sich auf eine relativ simple Methode: Stammbaumforschung (pedigrees). Der dabei zugrunde gelegte Gedanke war einfach: Wenn Begabung und Fähigkeit, Galton sprach vorwiegend von ability, ${ }^{13}$ durch

${ }^{9}$ Francis Galton, Hereditary Genius: An Inquiry into Its Laws and Consequences, London 21892, S. 340 (Erstausgabe 1869); auch zit. in DAVIE, Tracing the Criminal, S. 263.

10 Vgl. dazu auch Radzinowicz und Hood, The Emergence of Penal Policy, S.30; auch Henry Maudsley hatte den Endpunkt des Degenerationsprozesses in „sterile idiocy“ gesehen (MAUDSLEY, Physiology, S. 246f.).

11 Auch diese Idee lässt sich bei Darwin finden, ders., Descent of Man, London 1871, S. 177; hier spricht er die Befürchtung aus, dass wenn man nicht ,the reckless, the vicious and otherwise inferior members of society" daran hindern würde „from increasing at a quicker rate than the better classes of men, the nation will retrograde, as has occurred too often in the history of the world."

12 Siehe dazu Francis Galton, Hereditary Genius: An Inquiry into Its Laws and Consequences, London 1869 [Reprint London 1892], S. 1, wo er von ,selective breeding“ spricht, „quite practicable to produce a higly gifted race of men by judious marriage during several consecutive generations." Die Idee einer Reproduktionskontrolle über Menschen mit körperlichen oder geistigen Defekten war nicht neu, schon der Psychiater James Prichard hatte 1835 in seinem A Treatise on Insanity $(1835$, S. 336) Methoden thematisiert, wie man Heiraten unterer Schichten überwachen könne, um die Weitergabe von Idiotie und die erbliche Disposition vom Geisteskrankheiten unterbinden könnte; siehe dazu auch RADZINOWICZ und HOOD, Emergence of Penal Policy, S. 28.

13 Galton glaubte allerdings, dass ,ability' eine komplexe Eigenschaft sei, die auf mehreren erblichen Komponenten beruhe, die separat vererbt werden könnten, nämlich „capacity, zeal, and vigour": "for unless these three, or, at the very least, two of them, are combined, he [man, S.F.] cannot hope to make a figure in the world. The probability against inheriting a combination of three qualities not correlated together is necessarily in a triplicate proportion greater than it is against inheriting any one of them." Francis GaLton, Hereditary Genius: An Inquiry into Its Laws and Consequences, überarbeitete Aufl. New York 1879, S. 84; hier zit. nach GiLlHAM, Francis Galton, S. 163. 
die Natur bestimmt würden, dann müssten die nächsten Verwandten eines bedeutenden Mannes mit großer Wahrscheinlichkeit ebenfalls mit außergewöhnlichen Fähigkeiten ausgestattet sein. Frauen spielten in Galtons Stammbaumanalyse keine Rolle. Ihre ,Leistungen` waren seiner Ansicht nach nicht messbar, da sie in der Öffentlichkeit keine den Männer ebenbürtige Rolle spielten.

Der erste Artikel, in dem Galton seine Annahmen formulierte, erschien 1865 als zweiteiliger Beitrag über „Hereditary Talent and Character“ in Macmillan's Magazine, ${ }^{14}$ einer populären Zeitschrift, in der auch Matthew Arnold, Herbert Spencer, Thomas Henry Huxley und Charles Lyell publizierten. ${ }^{15}$ Dieser Aufsatz markiert den Beginn einer öffentlichen Diskussion über die Vererbung von Intelligenz, die bis heute andauert. ${ }^{16}$ Galton argumentiert darin, dass die allgemein anerkannte, durch die Natur bestimmte Weitergabe von Eigenschaften und Merkmalen bei Tieren in gleicher Weise bei Menschen angenommen werden müsse. Man müsse davon ausgehen, dass die Vererbung intellektueller Eigenschaften nach dem gleichen Mechanismus ablaufe wie die Vererbung physischer Merkmale, die bei der Züchtung von Haustieren oder Kulturpflanzen leicht zu beobachten sei. ${ }^{17}$ Schon Darwin hatte auf die durch gezielte Eingriffe erzeugten positiven Effekte bei Tierzüchtungen hinwiesen: „We cannot suppose that all the breeds were suddenly produced as perfect and as useful as we now see them; indeed in many cases, we know that this has not been their history. The key is man's power of accumulative selection: nature gives successive variations; man adds them up in certain directions useful to him. "18 Auf rein spekulativem Wege nahm Galton in seiner frühen Schrift sogar August Weismanns Keimplasmatheorie ${ }^{19}$ rund 20 Jahre vorweg, indem er behauptete, dass die Kontinuität des Keimplasmas (weibliche

${ }^{14}$ Francis Galton, Hereditary Talent and Character, in: Macmillan's Magazine 12 (1865), S. 157-166, S. 318-327.

${ }^{15}$ In Macmillan's Magazine erschienen auch Gedichte von Henry Wandsworth Longfellow und Christina Rossetti, vgl. Gillham, Francis Galton, S. 156.

16 Populäres Beispiel sind die Thesen von Thilo Sarrazin in seinem Buch Deutschland schafft sich $a b$ von 2009; zu den wissenschaftlichen Forschungsdiskussionen siehe z. B. das Interview „Es gibt kein Zentrum für unsere Intelligenz" mit dem amerikanischen Verhaltensgenetiker Robert Plomin, Professor am Zentrum für Soziale, Genetische und Entwicklungspsychiatrie am King's College in London, in: FAZ, Nr. 119, 24. Mai 2008, S. 35: „Vermutlich sind etwa 50 Prozent der intellektuellen Unterschiede zwischen den Menschen auf die Gene zurückzuführen. Das heißt allerdings auch, dass 50 Prozent nicht erblich sind. Neu ist für uns, dass der genetische Faktor im Laufe des Lebens sich verändert und im Alter eine zunehmend größere Rolle spielt.“

17 Erst spät, in seinen Memories of my Life (3. Aufl. London 1909, S. 288), zog Galton auch die Rolle des sozialen Umfeldes in Betracht, das für die Karrierechancen von Nachkommen entscheidend sein konnte. Ihm sei auch klar, so hieß es jetzt als Reaktion auf die vielfältigen Einwände auf seine Vorstellungen, dass der Sohn eines großen Mannes automatisch „will be placed in a more favourable position for advancement, than if he had been the son of an ordinary person."

18 Charles Darwin, On The Origin of Species by Means of Natural Selection, or the Preservation of Favored Races in the Struggle for Life, London 1859, S.29; hier zit. nach GILLHAM, Francis Galton, S. 156.

${ }^{19}$ Siehe dazu Kap. 5. 
und männliche Keimzellen) für die Weitergabe erworbener Eigenschaften wenig Raum lasse. ${ }^{20}$ Auch dieser Ansicht stimmte Charles Darwin zu: „I am inclined to agree with Francis Galton in believing that education and environment produce only a small effect on the mind of anyone, and that most of our qualities are innate." 21

Ausführlicher arbeitete Galton seine Überlegungen in der 1869 publizierten Untersuchung Hereditary Genius aus. Ihr lag eine größere Datenbasis zugrunde, und zum ersten Mal setzte er die Gaußsche Normalverteilungkurve (bell curve) als evaluierende Technik ein. ${ }^{22}$ Um nachzuweisen, dass Talent und Charakter erblich sind, sammelte er über Jahre Daten und Informationen berühmter und erfolgreicher Männer aus Lexika und Nachrufen der Times. Alle seine Berechnungen schienen mehr oder weniger das gleiche Ergebnis nahe zulegen: Die Nachkommen eminenter Männer zeigten eine größere Wahrscheinlichkeit, ebenfalls eine bedeutende Rolle zu spielen oder durch besondere Talente aufzufallen. Je näher die Verwandtschaft (Vater, Bruder, Sohn), desto größer diese Wahrscheinlichkeit. Die von ihm untersuchten 300 einflussreichen Familien zeigten eine deutlich höhere ability ratio als die Durchschnittsbevölkerung. Sie brachten bereits in der nächsten Generation fast 1000 Männer von Bedeutung und Einfluss hervor.

Galton war von seinen mathematischen Berechnungen aufgrund von Stammbaumanalysen so überzeugt, dass er sich gegen Ende seines Buches sogar berechtigt glaubte, seine Ergebnisse auf Rassen zu extrapolieren, obgleich ihm dafür gar keine vergleichbaren Daten zur Verfügung standen. Anlass zu seiner Übertragung gaben ihm lediglich die eigenen, während seiner Afrikareisen gemachten Erfahrungen. Diese hatten ihn davon überzeugt, dass die afrikanische Bevölkerung auf einer niedrigeren Stufe des Zivilisationsprozesses stehen geblieben sei und folglich auch weniger Intelligenz aufweise. ${ }^{23}$ Eine Rolle spielten dabei sicher auch Galtons enge Verbindungen zum Anthropologischen Institut ${ }^{24}$ und zur British

20 Siehe dazu Ruth Schwartz Cowan, Sir Francis Galton and the Study of Heredity in the Nineteenth Century, Ann Arbor/MI 1969, S.30f.; auch Gillham, Francis Galton, S. 157.

${ }^{21}$ Charles Darwin zitiert in: Karl Pearson, The Life, Letters and Labours of Francis Galton, Cambridge 1914, Bd. 1, S. 1, zit. auch in Gillham, Francis Galton, S. 155.

22 Galton, Hereditary Genius; zum genauen Verfahren siehe Gillham, Francis Galton, S. 157163.

${ }^{23}$ Ein Forscher, so schrieb Galton, „has to confront native chiefs in every inhabited place. The result is familiar enough - the white traveller almost invariably holds his own in their presence. It is seldom that we hear of a white Traveller meeting with a black chief whom he feels to be the better man." GaLton, Hereditary Genius, S.339; hier zit. nach Gilham, Francis Galton, S. 168.

24 Zur Anthropological Society of London (1863-1871) siehe ANGELIQue RichARDSON, Love and Eugenics in the Late Nineteenth Century: Rational Reproduction and the New Woman, Oxford 2003, S. 24, Anm. 99, die betont, dass hier bereits eine deutliche Verschiebung zur "new science of physical anthropology" stattgefunden habe, die sich auf eine "polygenic line“ konzentrierte im Gegensatz zur älteren "monogenic line“ der Ethnologie, die von der Abstammung eines Menschenprototyps (Adam und Eva) ausging. Diese neue physische Anthropologie habe argumentiert "for the fixity and persistence of racial characterists, transforming the concept of foreigness"; zur Fusion von Anthropological und Ethnological Society und die wachsende kulturelle Autorität von Wissenschaft siehe MARTIN FICHMAN, Biology and Politics: 
Association for the Advancement of Science, die eine eigene anthropologische Sektion unterhielt. Auf dem Weg zu einer systematischen Wissenschaft verlagerte sich die Tätigkeit der Anthropologie seit den frühen 1870er Jahren ganz auf die Beschreibung und Klassifizierung der unterschiedlichen körperlichen Merkmale von verschiedenen Rassen. Sie entwickelte sich zu einer physical anthropology, und Galton zeigte sich an der Quantifizierung physischer Attribute besonders interessiert. ${ }^{25}$ In jungen Jahren hatte er sich kurzzeitig sogar mit Phrenologie beschäftigt, die vor allem durch den schottischen Arzt George Combe popularisiert worden war, als wissenschaftliche Bewegung in Großbritannien aber völlig marginal blieb. ${ }^{26}$ Seit 1875 nahm Galton dann aber eine führende Position im Anthropometric and Racial Committee der British Association ein. ${ }^{27}$

Die Übertragung seiner Intelligenzforschung auf eine mögliche Rassenforschung, d.h. Galtons theoretische ,Sprünge' waren fester Bestandteil seines wissenschaftlichen Arbeitens und seiner Theoriebildung. Er war darin aber kein Einzelfall. Diskussionen über menschliches Verhalten, über Intelligenz, über Vererbung wurden lange Zeit von derartigen, heute methodisch unzulässigen Generalisierungen geprägt: „This temptation to leap from trying to understand and explain actual data to the grand and sweeping generalization whose basis derives only from personal observation and prejudice“, so gibt Nicolas Gillham zu bedenken, „has often been a hallmark of studies on genes, intelligence, and behaviour." ${ }^{28}$ Galtons zeitgenössische wissenschaftliche Leistung bestand aber zweifellos darin, dass er der Öffentlichkeit eine neue Theorie vorstellte, die zum ersten Mal von der strikten Erblichkeit intellektueller Fähigkeiten ausging. Daneben führte er mit der Stammbaumanalyse eine Methode ein, die, in dem was später human genetics genannt

Defining the Boundaries, in: Bernard Lightman (Hrsg.), Victorian Science in Context, Chicago und London 1997, S.94-118, hier S. 102: „the often acrimonoius disputes between the rival (anti-Darwinian) Anthropological Society and the (Darwinian) Ethological Society during the 1860s must be seen in the context of their mutual aim of establishing the paradigma of the scientific study of man'." Thomas Henry Huxley sei es schließlich 1871 geglückt, die beiden Gesellschaften zum Anthropological Institute of Great Britain and Ireland zusammenzuführen, wissenschaftlich gewann die ,physische Anthropologie für die nächsten Jahre den größeren Einfluss; zur Entwicklung der Anthropologie allgemein siehe ADAM KUPER, Anthropology, in: Theodore M. Porter und Dorothy Ross (Hrsg.), The Cambridge History of Science, Bd. 7: The Modern Social Sciences, Cambridge 2003, 354-378; zu den Programmen der Anthropologie siehe ders., The Invention of Primitive Society: Transformation of an Illusion, London 1988.

${ }^{25}$ DAVIE, Une des défigurations les plus tristes, S. 191-220.

26 Siehe dazu Gillham, Francis Galton, S.215-17; zur Geschichte der Phrenologie in Großbritannien siehe Roger Cooter, The Cultural Meaning of Popular Science: Phrenology and the Organisation of Consent in Nineteenth Century Britain, Cambridge 1984; DAVIE, Tracing the Criminal, S.37-42; Phrenologie blieb in Großbritannien eine marginale Erscheinung, als direkte Vorläuferin der psychiatrischen Debatten (Kapitel 3 dieser Arbeit) kann sie meines Erachtens nicht gelten, die medizinische Zunft nahm mehrheitlich eine skeptische Haltung ihr gegenüber ein, vgl. ElizABETH FeE, Nineteeth-Century Craniology: the Study of the Female Skull, in: Bulletin of the History of Medicine 53 (1979), S. 415-433.

27 Siehe DAVIE, Tracing the Criminal, S. 97.

${ }^{28}$ Vgl. Gillham, Francis Galton, S. 168. 
werden sollte, jahrzehntelang eine führende Rolle spielen sollte. Da Galton kein Biologe, sondern Mathematiker war, ${ }^{29}$ näherte er sich dem Problem der Vererbung nie, wie Gregor Mendel, auf dem Weg biologischer Experimente, sondern ausschließlich auf dem Weg quantitativer biostatistischer Analysen. ${ }^{30}$ In den späten 1870er und 1880er Jahren beschäftigte er sich dann intensiver mit der Entwicklung technischer Verfahren bzw. Methoden, die helfen sollten, seine eigenen Vermutungen über die Vererbung intellektueller Fähigkeiten zu bestätigen. Als physical anthropologist interessierte ihn dabei besonders der Zusammenhang zwischen geistiger Anlage und sichtbaren körperlichen Zeichen.

\subsection{Politik und Methode: composite photography}

Das Ende das Deportationssystems und das sogenannte ticket-of-leave-System, das eine Überwachung vorzeitig aus der Haft entlassener Strafgefangener vorsah, hatten in den 1850er und 1860er Jahren ,moralische Paniken' ${ }^{\text {31 }}$ in der Öffentlichkeit ausgelöst, die zur Verabschiedung des Habitual Criminals Act von 1869 und zwei Jahre später zu seiner modifizierten Version, dem Prevention of Crimes Act (1871), führten. Das Gesetz sah die Überwachung entlassener Strafgefangener durch Polizeikräfte vor. Bei Verdacht auf Rückfälligkeit lag die Beweislast der Unschuld beim ehemaligen Strafgefangenen. Dieses Überwachungssystem, so führen u. a. Clive Emsley und Leon Radzinowicz aus, funktionierte allerdings mehr schlecht als recht: "Supervision was not an English forte." ${ }^{32}$ Doch in einer Zeit, die durch starke Abwanderungsbewegungen vom Land in die Stadt und von Angst vor Überfremdung gekennzeichnet war, erhielt die Suche nach effizienten und verlässlichen Identifizierungsmethoden oberste Priorität und lenkte die Forschung in eine bestimmte Richtung. Simon Cole, der sich intensiv mit der Entwicklung kriminaltechnischer Erkennungsmethoden beschäftigt hat, beschreibt das Problem so: „There remained the thorny problem of how police men and magistrates were to recognise an ,habitual criminal' when they saw one, since there was evidence that many recidivists represented themselves as first offenders, and were dealt with as such in summary courts. " 33 Fraglos handelte es sich bei

29 Vgl. Gillham, Francis Galton, S.3; zunächst hatte Galton Medizin am King's College in London studiert, dieses aber zugunsten eines Mathematik-Studiums in Cambridge abgebrochen.

30 Vgl. dazu vor allem Francis Galton, Natural Inheritance (1888), und ders., Co-relations and Their Measurement, Chiefly from Anthropometric Data, Proceedings of the Royal Society 45 (1888), S. 135-45, in diesem Vortrag führte er die ersten Korrelationskoeffizienten für verschiedene Variablen ein.

$31 \mathrm{Zu}$ diesen ,moralischen Paniken' siehe Anm. 325 in Kap. 2.

32 Radzinowicz und Hood, The Emergence of Penal Policy, S. 250, Hervorhebung im Original.

33 Davie, Tracing the Criminal, S. 91; Radzinowicz und Hood, The Emergence of Penal Policy, S. 251; besonders aufschlussreich SIMON A. Cole, Suspect Identities: A History of Fingerprinting and Criminal Identification, Cambridge/Mass. 2001, S. 15: „The shift from classical to reformist jurisprudence [in the mid-nineteenth century, S.F.] demanded technologies of criminal identification." 
der Einführung photographischer Aufnahmen und dem Aufbau systematischer Verbrecherkarteien um Möglichkeiten, Wiederholungstäter besser erfassen und identifizieren zu können. Doch die Effizienz solcher Karteisysteme zeigte schon bald ihre Grenzen. Es war die schiere Masse an Fotomaterial, die eine rasche Bearbeitung zunehmend unmöglich machte bzw. die sich als so zeitaufwendig erwies, dass der Erfolg in keinem Verhältnis zur investierten Arbeit stand. Je größer die fotographischen Sammlungen bei Scotland Yard wurden, umso größer wurde auch die Wahrscheinlichkeit, dass Straftäter nicht identifiziert wurden. ${ }^{34}$

Edmund Du Cane, Vorsitzender der Directors of Convict Prisons und ab 1877 auch der Leiter der neu eingerichteten Prison Commission, wusste um die Defizite des polizeilichen Erkennungs- und Überwachungssystems. ${ }^{35}$ Die Schwierigkeiten einer effizienten Erfassung von Rückfalltätern thematisierte er 1875 in seiner Ansprache vor der repression-of-crime-Sektion der Social Science Association. Für ihn waren besonders die aktuellen - und das hieß zu diesem Zeitpunkt vor allem die medizinischen und psychiatrischen - Diskussionen über Kriminalitätsgenese und Verbrecherkonstitution unter verwaltungstechnischen Fragen von Bedeutung. ${ }^{36}$ Allerdings waren die Aussagen und Erkenntnisse des von ihm befragten Gefängnispersonals bezüglich der Anlage- und Umwelteinflüsse keineswegs eindeutig: Während Gefängnisgeistliche eher Umwelteinflüsse betonten, glaubten einige Gefängnisärzte eher an erbliche Dispositionen. ${ }^{37}$ Obwohl der Prison Commissioner die endgültige Beantwortung dieser Frage weiterer Forschung überlassen wollte, bekannte er sich persönlich zu der Auffassung, dass ein krimineller Lebenswandel sich an körperlichen Merkmalen ablesen lasse, die viele Straftäter miteinander teilten: „[I]t certainly is my own observation, that among a large number of criminals there are sure to be found a considerable proportion who have certain physical characteristics in common, which may or may not be attributed to the kind of life they lead, but which denote with certainty the habitual criminal. “38 Die Fotografien von Gefängnisinsassen hatten Du Cane darin bestä-

34 Zur Photographie als Ermittlungsmedium im Polizeidienst siehe u. a. MirosLaV VeC, Die Spur des Täters. Methoden der Identifikation in der Kriminalistik, Baden-Baden 2002; Peter BECKER, Dem Täter auf der Spur. Eine Geschichte der Kriminalistik, Darmstadt 2005; Susanne REGENER, Fotografische Erfassung. Zur Geschichte medialer Konstruktionen des Kriminellen, München 1999.

35 Edmund Du Cane (1830-1903), Lieutnant Colonel (Royal Engineers), Vorsitzender der Directors of Convict Prisons, Surveyor-General of Prisons und Inspector-General of Military Prisons, nach Schaffung der Prison Commission im Jahr 1877 auch ihr Vorsitzender bis 1895, DNB, 64 (1901), S. 528-29; ausführlich zu Du Cane siehe SÉAn Conville, The Social and Penal Ideas of Sir Edmund Du Cane, in: ders., English Local Prisons 1860-1900, London und New York 1995, S. 149-187.

36 Vgl. Du Cane, Address on Repression of Crime, bes. S. $303 \mathrm{f}$.

${ }^{37} \mathrm{Du}$ Cane selbst zeigte sich von Bruce Thomsons Aufsatz beeindruckt, obgleich dieser nicht den Einfluss der Umwelt berücksichtigte: „[S] howing in how many instances a great many members of the same family became criminals, and were even in prison at the same time, [...] does not prove any hereditary taint, but only that all the members are equally badly brought up and with the same result." Du CANE, Address on Repression of Crime, S. 302.

${ }^{38}$ Ebd., S. 302. 
tigt, dass es sich bei vielen von ihnen um Beispiele einer sich zu einem inferioren Typ zurückentwickelten ,Rasse' ( race) handelte:

The characteristics of this class are entirely those of the inferior races of mankind - wandering habits, utter laziness, absence of forethought or provision, want of moral sense, cunning, dirt, and instances may be found in which their physical characteristics approach those of the lower animals so that they seem to be going back to the type of what Professor Darwin calls ,our arboreal ancestors' ${ }^{\text {' }}$ '

Der Hinweis ist bedeutsam, da hier - ein Jahr vor dem Erscheinen von Lombrosos L'uomo delinquente - die Möglichkeit suggeriert wurde, dass es solche allen Kriminellen gemeinsamen äußeren Kennzeichen gab, die durch die Entwicklung eines effizienten Identifizierungsprogramms erfasst werden konnten. Offensichtlich gab es, wie Simon Cole anmerkt, eine innere Logik, die sich bei der Suche nach effizienten Identifizierungsmethoden mit Fotografie und der Vorstellung eines „criminal body“ 40 verband, den es zu dechiffrieren galt. Es sei eine unwiderstehliche Versuchung gewesen, Photographie über das hinaus zu nutzen, was sie zu leisten im Stande gewesen sei, d. h. sie nicht nur zur Erfassung und Registrierung von Straftätern einzusetzen, sondern die gemeinsamen physiognomischen Attribute bekannter Krimineller zu nutzen, um festzustellen, wie Kriminelle im Allgemeinen aussahen und dadurch zukünftige Verbrecher präventiv noch vor der Begehung einer Straftat zu identifizieren. ${ }^{41}$

Aus diesen Überlegungen heraus entstand die Zusammenarbeit zwischen dem unabhängigen Forscher Francis Galton und dem in staatlichen Diensten stehenden Prison Commissioner Edmund Du Cane. Letzterer verkörperte insofern einen neuen Typus des Beamten, als er sich nicht scheute, externe Expertise für verwaltungstechnische Aufgaben einzuholen und wissenschaftliche Projekte zu fördern. Was Du Cane mit Galton vormachte, würde sein Nachfolger im Amt, Sir Evelyn Ruggles-Brise, mit Galtons Schüler Karl Pearson in gewisser Weise wiederholen. ${ }^{42}$ Du Cane nahm zu Galton Kontakt auf, weil dieser sich durch sein Engagement im Anthropologischen Institut und durch die Erfassung und Verzeichnung körperlicher Merkmale bereits einen Namen gemacht hatte. In einem Brief an Galton gab Du Cane seiner Überzeugung Ausdruck: „Special forms of crime have a typical characteristic face; [... crimes of violence and crimes of lust certainly have. "43 Galtons positive Antwort auf die Anfrage, ob er sich anthropometrisch mit einer Gruppe von Insassen verschiedener convict-Gefängnisse, also Gefängnisse für Schwerverbrecher, beschäftigen wolle, wurde nicht nur durch sein Interesse an solchen Messungen bestimmt, sondern auch durch sein Interesse an Fragen der menschlichen Degeneration. Galton kannte die Arbeiten von Morel,

${ }^{39}$ Ebd., S. 302 f., Hervorhebung S.F.

40 Referenz zum Buchtitel von David G. Horns, The Criminal Body. Lombroso and the Anatomy of Deviance, New York und London 2003.

${ }^{41}$ Cole, Suspect Identities, S. 26; siehe auch DAvIE, Tracing the Criminal, S. 96.

${ }^{42}$ Siehe Kap. 5.

${ }^{43}$ Sir Edmund du Cane an Francis Galton, 12. Februar [1880 (?)], Galton Papers, 152/6A, University College London Archives, hier zit. nach DAVIE, Tracing the Criminal, S. 97. 
Thomson, Maudsley und Nicholson. ${ }^{44}$ Sowohl die Beziehung zwischen Kriminalität und Krankheit schien ihm ausgemacht als auch der Zusammenhang zwischen bestimmten körperlichen Kennzeichen und der Neigung ihres Trägers zu kriminellen Handlungen. ${ }^{45}$ Überzeugt davon, „[that] we are entitled to expect [...] in any large body of convicts a prevalence of the truly criminal characteristics, whatever these may be, " 46 ging er eifrig an die Arbeit, um eine Methode zu entwickeln, die diese Annahmen wissenschaftlich bestätigen konnte.

Dank Du Canes Unterstützung wurde es Galton ermöglicht, tausende, zum Zweck der Identifizierung im Home Office gelagerte Fotografien von Straftätern auszuwerten, sowie mit Strafgefangenen in den Gefängnissen und mit den entsprechenden Behörden zu sprechen. Er durfte Kopien der Fotos von ,verabscheuungswürdigen' Kriminellen (heinous criminals) für seine eigenen statistischen Untersuchungen anfertigen. ${ }^{47}$ Galton arbeitete vor allem mit Fotografien von Insassen der Gefängnisse Pentonville und Millbank, die durch persönliche Informationen über Leben und kriminelle Karriere der entsprechenden Straftäter ergänzt wurden. ${ }^{48}$ Auch bei dieser Analyse war die dahinter stehende Idee einfach: Wenn eine bestimmte Gruppe von Individuen bestimmte mentale Eigenschaften teilte, dann konnten die physiognomisch gemeinsamen Merkmale dadurch gefiltert und herausgearbeitet werden, indem Fotografien ihrer Gesichter übereinander gelagert wurden (composite photography). Dieses Verfahren würde, so Du Cane, die singulären, individuellen Merkmale in den Hintergrund und gemeinsame Merkmale verstärkt hervor treten lassen. Mit einem solcherart erzeugten kriminellen Durchschnittsgesicht, das die markanten Linien oder Merkmale des entsprechen-

44 Vgl. dazu Radzinowicz und Hood, The Emergence of Penal Policy, S. 29.

45 Neil Davie führt diese Ansicht Galtons auf den Einfluss des Arztes und Statistikers Dr. William Guy (1810-1885) zurück. Dieser hatte in seinem Buch Results of the Census of the Population of the Convict Prisons in England, taken in 1862 and 1873 (London 1875) auf der Grundlage des Zensus von 1862 Straftaten in 5 Gruppen unterteilt und ihre Häufigkeit mit vier Gruppen von männlichen Straftätern korreliert, die nach ihren mentalen und körperlichen Eigenschaften klassifiziert worden waren. Sein Ergebnis zeigte, dass Kriminelle, die an geistigen Störungen litten, besonders häufig in sexuelle Straftaten involviert waren, in versuchte Tötungen mit Gift und in Gewaltverbrechen, aber selten, wenig überraschend, in Verbrechen, die ein „maximum of forethought and contrivance“ benötigten, siehe dazu DAVIE, Tracing the Criminal, S.96f.; David Nicolson hat in seiner Präsidentenansprache von 1895 ähnliche Beziehungen zwischen mental deficiency und z. B. Gewaltverbrechen angesprochen, allerdings diente ihm dieser Zusammenhang dann zu Demonstration, dass die statistisch stets viel größere Zahl von Eigentumsdelikten eben nicht von geistig beschränkten Personen begangen wurden, siehe NiCOLSON, Crime, Criminals, S. 587.

46 Francis Galton, Address to the Department of Anthropology of the British Association, in: Nature 16 (23. August 1877), S. 346.

47 Francis Galton, Address to Section D - Biology (Department of Anthropology), British Association for the Advancement of Science, Plymouth, in: Nature 16 (23. August 1877), S.344347, hier S. 346.

48 Siehe Davie, Tracing the Criminal, S. 97 (Home Office an Francis Galton, 18. April-22 May 1877, University College London Archives, Galton Papers, 158/1B); siehe auch FranCIS GALTON, Composite Portraits, in: Journal of the Anthropological Institute 8 (1878), S. 132-144, hier S. 143. 
den Verbrechertypus hervorhob, ${ }^{49}$ sollten dann Aufnahmen mutmaßlicher Verbrecher abgeglichen werden, um erste Anhaltspunkte darüber zu gewinnen, ob es sich bei der entsprechenden Person um einen kriminellen Charakter handelte oder eher nicht. Unterteilt wurden die Photographien von Gefängnisinsassen nach der Natur ihrer Straftaten in drei Gruppen. Die erste Gruppe umfasste Mord, Totschlag und Einbruch, die zweite schwere Verbrechen (felony) und Fälschung (forgery), die dritte sexuelle Straftaten.

Ein Jahr nach seinem Bericht über die composite photography vor der British Association erläuterte Galton seine Methode in einem großen Abendvortrag im Anthropologischen Institut. ${ }^{50}$ Er konnte bis zu acht Fotografien übereinander lagern, alle Fotos mussten die gleiche Größe haben, und alle Personen die gleiche Haltung einnehmen: Damit ließe sich der einzigartige Ausdruck von Gewaltverbrechern isolieren, so behauptete er. ${ }^{51}$ Allerdings wurde Galton in der Folgezeit zunehmend bewusst, dass man den kriminellen Typus so nicht erschließen konnte. Das Problem war, dass die Durchschnittskonturen solcherart kombinierter Gesichter ein relativ gleichmäßiges Gesicht erzeugten, das fast schön zu nennen war, denn es ignorierte alle extremen Abweichungen individueller Gesichter. Spätestens in seinen Inquiries into Human Faculty and its Development, die 1883 erschienen, musste Galton zugeben, dass zusammengesetzte Fotografien „produce faces of mean description, with no villainy written on them. "52 Zwar unternahm er noch einmal den Versuch zusammengesetzter Fotografien von Geisteskranken, aber deren Gesichtszüge waren so unregelmäßig, dass das Hervortreten einer klaren gemeinsamen Linie unmöglich war. ${ }^{53}$

In den Behörden erzeugten Galtons Experimente mit composite photography keine weiteren Folgen. Die Ergebnisse wurden einfach ad acta gelegt. Was an dieser Episode aber aufschlussreich erscheint, ist nicht nur Galtons eigenes Bestreben, eine akzeptable Methode zu entwickeln, die seine allein aus subjektiver Wahrnehmung gewonnenen Ansichten als richtig bestätigen sollte, sondern auch die enge Verflechtung von wissenschaftlicher Methodenentwicklung und politischem Zeitgeist. Wachsende Überfremdungsängste hatten die Suche nach effizienten Identifikationsmethoden sicherlich angeheizt. Die allgemeine politische Verunsicherung kam in England z. B. auch das harsche Vorgehen gegen Wohnsitz-

${ }^{49}$ Für die Details dieser Technik siehe FranCIS GaLton, Inquiries into Human Faculty and its Development, London 1907, Appendix 1, S. 221-241; zum Kontext am besten DAVIE, Une des défigurations les plus tristes, S. 191-220; auch Peter Hamilton und Roger Hargreaves, The Beautiful and the Damned: The Creation of Identity in Nineteenth Century Photography, London 2001, bes. Kap. 3; DAVID GreEN, Veins of Resemblance: Photography and Eugenics, in: The Oxford Art Journal 7 (1985), S.3-16; Allan Sekula, The Body and the Archive, in: October 39 (1986), S. 3-64.

50 Francis Gatton, Composite Portraits, in: Journal of the Anthropological Institute 8 (1878), S. 132-144.

${ }^{51}$ Vgl. dazu FranCis GaLton, in: Nature (1877), S. 346.

52 Galton, Inquiries into Human Faculty and Its Development, zit. nach GillHam, Francis Galton, S. 217.

${ }^{53}$ Siehe dazu Gillham, Francis Galton, S. 219. 
lose und Landstreicher zum Ausdruck. ${ }^{54}$ Die Erkennungsmethoden konnten nur dann funktionieren, wenn äußerlich sichtbare und damit erkennbare Merkmale, die den Kriminellen vom Nicht-Kriminellen unterschieden, tatsächlich existierten. Das Bedürfnis nach dem Vorhandensein solcher Merkmale wurde getragen von der Hoffnung, man könne bald besser und genauer einschätzen, mit wem man es zu tun habe. In den besorgten Mittel- und Oberschichten hätte dies zweifellos zu einer gewissen Beruhigung beigetragen.

\subsection{Daktyloskopie}

Für Galton als Wissenschaftler war es bezeichnend, dass er sich durch den Fehlschlag der composite photography nicht veranlasst sah, seine Hypothese von der grundsätzlichen Andersartigkeit von Kriminellen zu überdenken. Während seine Beschäftigung mit composite photography nicht in die Annalen der Kriminalgeschichte eingehen sollte, war einer anderen Erfindung langfristig ein Erfolg sicher. Wieder ging der Anstoß von seinem Cousin Charles Darwin aus. Dieser hatte im Frühjahr 1880 den Brief eines jungen schottischen Arztes, Henry Faulds vom Tsukiji Hospital in Tokio, erhalten, der sich seit 1878 mit Fingerabdrücken zunächst auf alten prähistorischen Keramikvasen, dann mit denen von Affen und schließlich mit Fingerabdrücken zeitgenössischer Japaner beschäftigt hatte. ${ }^{55}$ Faulds glaubte, dass seine Entdeckung von einigem Interesse für den von ihm sehr bewunderten Darwin sein würde: Nicht nur waren die Linien der Abdrücke einzigartig für jedes Individuum, auch klassifizierende Einordnungen ließen sich vornehmen. Die Brauchbarkeit dieser Technik zur Identifizierung von Kriminellen deutete Faulds bereits in seinem Brief an Darwin an. Darwin sandte diesen Brief an Galton weiter, der ihn im Anthropologischen Institut vorstellen sollte. Als eine Veröffentlichung dieses Briefes durch das Institut nicht zustande kam, veröffentlichte Faulds seine Erkenntnisse in der Zeitschrift Nature. Er hob darin die Ähnlichkeit menschlicher Fingerabdrücke mit denen von Affen hervor, die weiter untersucht werden sollte. Doch ein anderer, von ihm formulierter Vorschlag erregte weitaus größere Beachtung: „When bloody finger-marks or impressions on clay, glass, \&c., exist, they may lead to the scientific identification of criminals. "56 Faulds zog auch die forensische Identifikation von Opfern krimineller Straftaten in Betracht. Selbst wenn nur die Hände verstümmelter Leichen geborgen werden konnten, könne Vererbung Experten in die Lage versetzen, mit beachtlicher Wahrscheinlichkeit die Verwandten des Verstorbenen zu bestimmen, in manchen Fällen sogar mit höchster Sicherheit. ${ }^{57}$

\footnotetext{
54 Siehe dazu Radzinowicz und Hood, The Emergence of Penal Policy, Kap.11: Draining the Reservoir of Crime: The Vagrant Criminal, S. 339-375.

55 Zum Folgenden besonders das Kapitel ,Fingerprinting' in: Gillham, Francis Galton, S.231249.

${ }^{56}$ Henry Faulds, On Skin Furrows in the Hands, in: Nature 22 (1880), S. 605.

57 Vgl. ebd.
} 
Bereits in den 1860er Jahren hatte sich ein anderer britischer Staatsbürger, Sir William Herschel, mit Fingerabdrücken im fernen Indien beschäftigt. Als Verwaltungsbeamter in Bengalen war Herschel mit der Abwicklung von Pensionszahlungen an die in britischen Diensten stehenden Inder betraut. Aufgrund des hohen Anteils an Analphabeten und zahlreicher Versuche von Einheimischen, unberechtigte Ansprüche auf britische Pensionszahlungen zu erheben, war Herschel dazu übergegangen, Verträge und Dokumente mit Fingerabdrücken unterzeichnen zu lassen, nachdem durch Zufall ein Einheimischer seine Hand vor Vertragsunterzeichnung in Öltinte gesteckt und dadurch ein deutliches Zeichen auf dem Dokument hinterlassen hatte. Herschel erkannte schnell, wie nützlich Fingerabdrücke zu Identifizierung von Personen sein konnten. Nach seiner Rückkehr nach Oxford veröffentlichte er am 25. November 1880, genau eine Ausgabe später als Faulds, seine Erkenntnisse in Nature. ${ }^{58}$ Er verwies auf seine 20jährige Praxis mit Fingerabdrücken und - ein wichtiger Hinweis - bemerkte, dass sich Fingerabdrücke nicht veränderten, die typischen Linien und Kurven ein Leben lang erhalten blieben und sich sogar nach Verletzungen der Oberfläche wiederherstellten. Nicht nur zur Identifikation von Deserteuren und Kriminellen glaubte er ein passendes System gefunden zu haben, sondern auch zur Klärung verwandtschaftlicher Beziehungen, z. B. von legitimen oder illegitimen Erben, einem Problem, das den Zeitgenossen seit dem Aufsehen erregenden Tichborne-Fall lebhaft vor Augen stand. ${ }^{59}$

Galtons wissenschaftliche Leistung bestand nun darin, dass er ein Klassifikationssystem mit Ein- und Unterteilungen, mit Verhältnisbestimmungen von Linien, ihrem Kurvenverlauf und ihren Zwischenräumen ausarbeitete. Mit Hilfe dieses Systems sollte es möglich werden, nicht nur eine systematische Kartei aufzubauen, sondern auch den Abgleich mit unbekannten, sichergestellten Fingerabdrücken möglichst rasch zu gewährleisten. Galton analysierte über 2500 Fingerabdrücke, die er von Besuchern seines Anthropometrischen Labors in South Kensington über Jahre hin angefertigt hatte. ${ }^{60}$ Ende der $1880 \mathrm{er}$ Jahre beeindruckte er

58 William J. Herschel, Skin Furrows of the Hand, in: Nature 23 (1880), S. 76.

59 Sowohl Faulds als auch Herschel spielten auf den berühmten Tichborne-Fall an, einen Prozess um Betrug und angebliche Identität eines verschollenen reichen Erbens, der im viktorianischen England großes Aufsehen erregte. Auch Galton bemerkte im Januar 1888 in seiner Präsidentenansprache im Anthropologischen Institut, dass ein solch kostspieliger und langwieriger Prozess mit der entsprechenden Technik und unter der Voraussetzung, man wäre im Besitz eines Fingerabdrucks des echten Tichborne gewesen, hätte vermieden werden können, siehe Francis Galton, Presidential Address, January 1888, in: Journal of the Anthropological Institute 17 (1888), S.35; zum Fall Tichborne siehe Edgar Feuchtwanger, Der Fall Tichborne, in: Andreas Fahrmeir und Sabine Freitag (Hrsg.), Mord und andere Kleinigkeiten. Historische Kriminalfälle aus fünf Jahrhunderten, München 2001, S. 144-168.

60 Galton hatte sein Anthropometrisches Labor während der Internationalen Gesundheitsausstellung 1884/85 in South Kensington eingerichtet. Später wurde es am University College in London untergebracht. Galton führte hier neben seinen biometrischen Messungen auch die ersten Intelligenztests mit Kindern durch, siehe MaURICE BRIDGELAND, Pioneer Work with Maladjusted Children. A Study of the Development of Therapeutic Education, London 1971, S. 49; Francis Galton, The Anthropometric Laboratory, in: The Fortnightly Review 31 (1882), S. 332-338. 
dann die Zuhörer der Royal Society mit seinen Vorträgen über sein System. ${ }^{61}$ Wie gewöhnlich vermittelte Galton seine Erkenntnisse auch in anderen populären Medien, diesmal durch einen Artikel für die Zeitschrift Nineteenth Century über „Identification by Finger-Tips“. ${ }^{2}$ In ihm beschrieb er Fingerabdrücke als „visible token of identity“, 63 brauchbar für Armee, Gefängnis und - seiner Zeit weit voraus - für die Reisepässe unbescholtener Bürger. Und er beschwor eine Zeit, „when every convict shall have prints taken of his fingers by the prison photographer, at the beginning and end of his imprisonment, and a register made of them. "64

Galton hatte auch bei diesen Experimenten nie sein anthropologisches Interesse aus den Augen verloren. Um herauszufinden, ob Rassenunterschiede in Fingerabdrücken sichtbar wurden, ließ er sich von Schulleitern in London, Cardiff und Niger Fingerabdrücke ihrer Schüler schicken. Das ermöglichte ihm den Vergleich von englischen, walisischen und afrikanischen Schulkindern. ${ }^{65}$ Was dabei herauskam, musste ihn jedoch erneut enttäuschen: „there is no peculiar pattern which characterises persons of the above races. "66 Allerdings ließ sich Galton in diesem Zusammenhang zu einer Bemerkung verleiten, die mehr über ihn als über die untersuchten Objekte verriet: „Still, whether it be from pure fancy on my part, or from some real peculiarity, the general aspect of the Negro print strikes me as characteristic. The width of the ridges seems more uniform, their intervals more regular, and their courses more parallel than with us. In short, they give an idea of greater simplicity, due to causes that I have not yet succeeded in submitting to the test of measurement."67 Indem Galton die Fingerabdrücke von Schwarzafrikanern als einfacher und weniger komplex beschrieb, evozierte er das Bild des primitiven Farbigen, dessen beschränkte mentale Kapazitäten sich in der Einfachheit seiner Fingerabdrücke widerspiegelten. Aber am Ende sah sich der gentleman scientist doch gezwungen zuzugeben, dass die Intelligenz einer Person nicht in ihren Fingerabdrücken zum Ausdruck kam: „I have prints of eminent thinkers and of eminent statesmen that can be matched by those of congenital idiots. No indications of temperament, character, or ability can be found in finger marks, so far as I have been able to discover." 68

Im Gegensatz zur composite photography hatte die Entwicklung der Daktyloskopie allerdings administrative Folgen. Die Resonanz auf Galtons 1892 publiziertes

${ }^{61}$ Francis Galton, The Patterns in Thumb and Finger Marks - On the Arrangement into Naturally Distinct Classes, the Permanence of the Papillary ridges that make them, and the Resemblance of their Classes to Ordinary Genera, in: Philosophical Transactions of the Royal Society of London, Series B 182 (1891), S. 1-23; ders. The Patterns in Thumb and Finger Marks, in: Proceedings of the Royal Society 48 (1890), S. 455-457; ders., Method of Indexing Fingerprints, in: Proceedings of the Royal Society 54 (1891), S. 540-545.

62 Francis GaLton, Identification by Finger-Tips, in: Nineteenth Century 30 (1891), S. 303-311.

63 Ebd., S. 303.

${ }^{64}$ Ebd., S. 311.

${ }^{65}$ Vgl. dazu Gilham, Francis Galton, S. 242.

66 GaLton, Finger Prints, London 1892, S. 192f., Hervorhebung im Original.

${ }^{67}$ Ebd., S. 196, Hervorhebung S. F.

${ }^{68}$ Ebd., S. 197; das Zitat findet sich auch bei DAVIE, Tracing the Criminal, S. 196, und ForREST, Francis Galton, S. 217; zur großen Resonanz des Buches siehe GILLHAM, Francis Galton, S.243f. 
Buch Finger-Prints ${ }^{69}$ führte zur Einberufung einer Untersuchungskommission des Innenministeriums, die sich im Oktober 1893 unter Vorsitz des Permanent UnderSecretary Edward Troup mit den Vorzügen des Erkennungssystems beschäftigte. ${ }^{70}$ Das Komitee ließ sich die Methode von Galton in seinem Anthropometrischen Labor demonstrieren und zeigte sich beeindruckt. Aber es war Galton selbst, der bei dieser Gelegenheit auf einige Schwierigkeiten hinwies, die eine zweifelsfreie Identifizierung erschwerten. Zustande kam schließlich ein Kompromiss, in dem das Komitee die Kombination aus Daktyloskopie und der aus Frankreich übernommenen Anthropometrie Alphonse Bertillons empfahl. Erst das Belper-Komitee sprach sich 1899 für die Daktyloskopie als der eindeutig besseren Methode zur Identifizierung Krimineller aus, nachdem ihre Klassifizierung eine Weiterentwicklung durch Sir Edward Henry erfahren hatte. Dieser nahm im Mai 1901 einen Posten als Assistant Commissioner im Criminal Investigation Department von Scotland Yard auf, wo im Juli 1901 die Central Fingerprint Branch eingerichtet wurde. ${ }^{71}$ Wenig später kam es zum ersten Indizienprozess, in dem die mutmaßlichen Mörder von Depthford aufgrund von Fingerabdrücken überführt wurden. ${ }^{72}$

\subsection{Differentielle Fertilität und Vererbung}

Galton mochte der Erfolg der Daktyloskopie schmeicheln. Was seine eigenen Ambitionen und Interessen betraf, brachte sie ihn aber nicht wirklich weiter. Fingerabdrücke waren in dem Sinne neutral und objektiv, als sie weder Aufschlüsse über die Hautfarbe oder das Geschlecht einer Person noch über deren kriminelle Dispositionen zuließen. Sie blieben ein technisches Werkzeug zur Überführung von Straftätern, mehr aber auch nicht. Mochte die Polizei mit dieser Methode die ersehnte Professionalität durch technischen Fortschritt demonstrieren können, für einen Nachweis der Vererbung intellektueller Fähigkeiten, wie Galton ihn anstrebte, taugte sie nichts. ${ }^{73}$ Galtons Interesse an der Anthropometrie und seine Experi-

69 Siehe Francis Galton, Finger Prints (1892); ders., Finger Print Directories, London 1895; siehe auch Francis Galton, Personal Identification and Description, in: Nature 38 (1890), S. 201; STEPHEN M. STIGLER, Galton and Identifikation by Fingerprints, in: Genetics 140 (1995), S. 857-860.

70 Siehe zum Folgenden ausführlich AnNe M. JosEPH, Anthropometry, the Police Expert, and the Deptford Murders. The Contested Introduction of Fingerprinting for the Identification of Criminals in Late Victorian and Edwardian Britain, in: Jane Caplan und John Torpey (Hrsg.), Documenting Individual Identity. The Development of State Practices in the Modern World, Princeton/NJ u. a. 2001, S. 164-183.

71 Siehe Gillham, Francis Galton, S. 249.

$72 \mathrm{Zu}$ diesem Fall siehe JosEPH, Anthropometry, S. 164-184.

73 Siehe Cole, Suspect Identity, S. 100: „Fingerprint examiners strengthened their authority by disassociating themselves from their colleagues who speculated about predictive powers of fingerprints to tell, not only the past, but also the future. By turning the fingerprint into an empty signifier - a sign devoid of information about a body's race, ethnicity, heredity, character, or criminal propensity - fingerprint examiners made fingerprint examination seem less value-laden, more factual [...] [A]ny correlation of fingerprint patterns with race, heredity or criminal propensity would have been dangerous to the credibility of forensic fingerprint identification." 
mente sowohl mit der composite photography als auch mit Fingerabdrücken, so urteilt Neil Davie, „can all be seen as part and parcel of his eugenics based desire to find what he considered to be an objective method to sort out the sheep from the goats, to determine by means of mental and physical measurement just who were the members of this degenerate underclass. " 74 Wo er Mitglieder dieser degenerierten Unterklasse, zu denen Galton auch Kriminelle zählte, finden konnte, galt Galton längst als ausgemacht: in den Asylen, Gefängnissen und Hospitälern des Landes.

Wie bereits erwähnt, näherte sich Galton dem Problem der Vererbung auf mathematischem Wege durch Berechnung des Auftretens von Phänotypen, nicht durch biologische Experimente an, die erst um 1900 nach der Wiederentdeckung der Mendelschen Vererbungsgesetze vom wohl bekanntesten englischen Biologen William Bateson unternommen wurden. Dieser sah durch das Züchten einfacher Organismen und deren Kreuzung eine wissenschaftliche Möglichkeit, zu gesicherten Aussagen über den Vererbungsmechanismus zu gelangen. ${ }^{75}$ Galton dagegen wandte sich ab den 1880er Jahren verstärkt dem Ausbau der biometrischen Statistik zu, um Wahrscheinlichkeiten der selektiven Reproduktion und dem Auftreten von Varianz innerhalb einer Art auf die Spur zu kommen. 1888 erschienen dazu seine zwei wichtigsten Publikationen: das Buch Natural Inheritance und ein Artikelbeitrag in der Schriftenreihe der Royal Society. ${ }^{76}$ Natural Inheritance enthielt seine gesamten bis dahin angestellten Überlegungen zum Vererbungsproblem. Es war rein theoretisch angelegt, denn es ließ zwar unter Berechnung von Korrelationskoeffizienten auf der Basis von Massendaten Aussagen über Vererbungsverhältnisse und -wahrscheinlichkeiten $\mathrm{zu}$, aber stellte keinerlei erklärende Modelle bereit. Galton wusste zwar von Chromosomen und deren Teilung, aber ihre Funktion war ihm völlig unbekannt. ${ }^{77}$ Er entwickelte auf diese Weise, so hat es Nicholas Gillham treffend formuliert, „the second best theory of heredity “ ${ }^{78}$

Während Galton sich verstärkt der korrelativen Statistik zuwandte, erlebte die von ihm in den 1860er Jahren entwickelte Stammbaumanalyse und die Frage nach der Vererbung von Intelligenz durch das veränderte politische Klima der 1880er und 1890er Jahre einen bemerkenswerten Aufschwung. Die wachsende Sorge über die europaweit diskutierten Degenerationserscheinungen trug dazu

74 Davie, Tracing the Criminal, S. $245 \mathrm{f}$.

75 Siehe dazu ausführlicher Kap. 5 dieser Arbeit; siehe auch William BATESON, Mendel's Principles of Heredity, Cambridge 1909; zur Wiederentdeckung der Mendelschen Gesetze durch drei unabhängig voreinander arbeitete Biologen um 1900, siehe DonALD MACKENZIE, Sociobiologies in Competition: The Biometrician-Mendelian Debate, in: Charles Webster (Hrsg.), Biology, Medicine, and Society, 1840-1940, Cambridge 1981, S. 243-288, hier S. 244.

76 Francis Galton, Natural Inheritance, London 1889; Francis Galton, Co-relations and Their Measurement, Chiefly from Anthropometric Data, in: Proceedings of the Royal Society 45 (1888), S. 135-145.

77 Siehe dazu Gillham, Francis Galton, S. 258f.

78 Ebd., S. 259. Galtons theoretische Ergebnisse sollten dabei so konsistent wie möglich mit den experimentellen Beobachtungen sein; auch in diesem Kontext ging es stets nur um angeborene, nicht um erworbene Eigenschaften. 
bei, dass Hereditary Genius in seiner zweiten englischen Auflage im Jahr 1892 auf eine weitaus größere öffentliche Resonanz stieß als noch 20 Jahre zuvor. ${ }^{79}$ Galton habe auf eindrucksvolle Weise demonstriert, so schrieb The Nation zur Neuauflage, dass Kinder nicht mit den gleichen geistigen Fähigkeiten geboren würden und Unterschiede in Intellekt und Begabung nicht auf individuelle Schulung, Erziehung und soziales Umfeld zurückzuführen seien, sondern „that individuals inherit different intellectual capacities.“ Das Fazit müsse deshalb lauten: „nature limits the powers of the mind as definitely as those of the body. " 80

Die Frage von Intelligenz und ihrer Erblichkeit spielte eine wichtige Rolle im Kontext der Schuluntersuchungen von Kindern und Jugendlichen seit Einführung der allgemeinen Schulpflicht 1880. Die zentrale Frage war dabei, wie mit geistig behinderten oder zurückgebliebenen Kindern verfahren werden sollte und welcher gesetzlichen Regelungen es bedurfte, um Kinder in Spezialschulen unterbringen zu können. Kinderwohlfahrtsverbände und Leiter von speziellen Schulen für mentally defective children forderten staatliche Unterstützung für den Ausbau ihrer eigenen Institutionen. Auf privaten Druck hin wurde 1904 die Royal Commission on the Care and Control of the Feeble-Minded ins Leben gerufen, die vier Jahre später ihre Ergebnisse der Öffentlichkeit vorlegte. ${ }^{81} \mathrm{Zu}$ den Gutachtern dieser Kommission gehörten vor allem Experten für mental deficiency wie der Psychiater und Eugeniker Alfred Tredgold und der Fachmann für Intelligenzfragen Francis Galton. Wenig überraschend vertraten die meisten von der Kommission angehörten Experten die Auffassung, dass geistige Behinderungen und Intelligenzdefizite in einem starken Maße, bis zu 90 Prozent, erblich bedingt seien. Als Folgen der geistigen Defizite benannten sie die Tendenz zu unkontrollierter, überproportionaler Fertilität, die vor allem der Promiskuität geistig behinderter Frauen zugeschrieben wurde, ${ }^{82}$ und die Neigung zu anti-sozialem und kriminellem Verhalten. Galton selbst sprach in diesem Zusammenhang nicht von einem angeborenen kriminellen Gen, wohl aber von angeborenen geistigen Defiziten, die kriminelle Dispositionen begünstigten. Unkontrolliert und sich selbst überlassen, so erklärte Galton dem Untersuchungsausschuss, würde das soziale Absinken dieser intellektuell unterlegenen oder geistig behinderten Menschen in kriminelle Lebensweisen durch deren „absence of will-power to resist temptation“83 kaum aufzuhalten sein. Nur konsequente Einweisung in Heime und Asyle könne hier einen Riegel vorschieben.

${ }^{79}$ Es gab auch eine überarbeitete amerikanische Ausgabe aus dem Jahr 1879.

80 The Nation, 6. April 1893; zit. nach Gillham, Francis Galton, S. 171.

${ }^{81}$ Eine gute Analyse über die Tätigkeit dieser Kommission findet sich in Thomson, Mental Deficiency, S. 23-33.

82 Während ,normale` Paare im Schnitt vier Kinder zur Welt brachten, waren es bei, defekten ‘ Paaren sieben, wie Alfred Tredgold, Verfasser des in vielen Auflagen gedruckten Standardwerks Mental Deficiency, vorrechnete, siehe dazu DAVID BARKER, The Biology of Stupidity: Genetics, Eugenics and Mental Deficiency in the Inter-War Years, in: British Journal for the History of Science 22 (1989), S. 347-375, hier S. 349.

${ }^{83}$ Francis Galton, Segregation, in: The Problem of the Feeble-Minded: An Abstract of the Royal Commission on the Care and Control of the Feeble-Minded, London 1909, S. 81-85. 
Die Vorstellung einer überproportionalen Vermehrung geistig defizitärer Personen gehörte in einen weiter ausgreifenden gesellschaftlichen Diskussionszusammenhang, der sich zeitgleich auch in Frankreich und Deutschland beobachten ließ: Es war die Sorge über eine differential birth rate. Darin wurde die Vermutung ausgesprochen, die unteren Schichten würden sich besonders rasant und unkontrolliert vermehren, während in den Mittel- und Oberschichten ein bedrohlicher Geburtenrückgang zu verzeichnen sei ${ }^{84}$ Aufgrund der Vorstellung erblicher Intelligenz würden die intellektuellen Fähigkeiten in der Gesamtbevölkerung sinken und an ihre Stelle unerwünschte Eigenschaften und Dispositionen im Überfluss auftreten: „The weaker the Intellect [...] the greater appears to be the strength of the reproductive faculties" 85 brachte die alarmierte Sozialreformerin Mary Dendy diese Überzeugung in einem Brief an Galton auf den Nenner. Es war allerdings der Galton Schüler Karl Pearson, ein hochbegabter Mathematiker und Statistiker, der 1903 in seiner Huxley Lecture im Anthropologischen Institut aufzeigte, dass 25\% der Eltern 50\% der nächsten Generation produzierten. ${ }^{86}$ Während die von ihm untersuchten Klassen 1 und 2 (Ärzte, Wissenschaftler, Geistliche, Bankiers) eine Geburtsrate von 3.4 Kindern aufwiesen, errechnete er für die Arbeiterschicht eine Durchschnittsrate von 6.1 Kindern pro Familie. Pearsons Interpretation ließ nur einen Schluss zu: „The mentally better stock in the nation is not reproducing itself at the same rate as it did of old; the less able and the less energetic are more fertile than the better stocks. " ${ }^{87}$ Tatsächlich basierten Pearsons Berechnungen auf höchst fragwürdigem Zahlenmaterial, denn bis 1911 wurden schichtspezifischen Details in den Erhebungen gar nicht erfasst. ${ }^{88}$ Zudem waren die Sterblichkeitsraten von Kindern aus unterschiedlichen Schichten nicht berücksichtigt. ${ }^{89}$

Doch der Sorge um einen racial decline wurde durch das Bekanntwerden des Burenkriegdebakels (1899-1902) ein enormer Schub versetzt. Großbritannien hatte drei Jahre und 250 Millionen Pfund für einen Krieg aufgewendet, den das Land offenkundig nicht eindeutig zu gewinnen in der Lage war. Die hohe Zahl an Ausmusterungen, drei von fünf Bewerbern für den Militärdienst wurden aufgrund

84 Siehe Simon Szreter, Fertility, Class and Gender in Britain 1860-1940, Cambridge 1996; Richard A. Soloway, Demography and Degeneration. Eugenics and the Declining Birthrate in Twentieth-Century Britain, Chapel Hill und London 1995, bes. S.3-18; Geoffrey R. SEArLe, Eugenics: The Early Years, in: The Galton Institute (Hrsg.), Essays in the History of Eugenics, London 1998, S. 25.

85 Mary Dendy an Francis Galton, Februar 1909, hier zit. nach KeLves, In Search of Eugenics, S. 107, auch zit. in Davie, Tracing the Criminal, S. 246.

86 Vgl. Radzinowicz und Hood, The Emergence of Penal Policy, S.30; Kelves, Eugenics, S. 74; SOLOWAY, Demography, S. 12f.

87 Vgl. dazu KarL PEARSON, On the Inheritance of the Mental Characters in Man, and Its Comparison with the Inheritance of the Physical Characters, in: Journal of the Anthropological Institute 33 (1903), S. 179-237.

88 Siehe Greta Jones, Theoretical Foundations of Eugenics, in: The Galton Institute (Hrsg.), Essays in the History of Eugenics, London 1998, S. 1-19, hier S. 11.

89 Siehe SolowaY, Demography and Degeneration, S. 8. 
körperlichen Ungenügens abgewiesen, ${ }^{90}$ ließ erneut die Sorge über eine physische Verschlechterung bestimmter Klassen in der Bevölkerung aufkommen und zugleich die Suche nach Mitteln dringlich erscheinen, durch welche dieser Prozess am effektivsten reduziert werden konnte. ${ }^{91}$ Die Frage, wie Großbritannien künftig den Bedarf an tauglichen Rekruten decken sollte, verband sich mit der Sorge, wie das Land mit den aufstrebenden Industrienationen USA und vor allem Deutschland noch Schritt halten konnte. Biologistische Erklärungen, insbesondere Degenerationstheorien, konnten in einem solchen Klima gut gedeihen, obgleich die offiziellen Behörden diesen Mutmaßungen entschieden entgegen traten.

Das 1903 einberufene Inter-Departmental Committee on Physical Deterioration, das sich nicht aus Eugenikern, Intelligenz-Experten wie Galton und Leitern von Spezialschulen für geistig Behinderte zusammensetzte, sondern aus Vertretern des Innenministeriums und lokaler Verwaltungsstellen, ließ besonders Mediziner, Erzieher, Lehrer und Sozialarbeiter zu Wort kommen. Diese stellten den schlechten Gesundheitszustand der unteren Schichten nicht in Abrede, waren aber mehrheitlich der Auffassung, dass Unter- und Mangelernährung, die katastrophalen Wohnbedingungen, Alkoholismus, elterliche Vernachlässigung und unzureichende medizinische Versorgung dafür verantwortlich gemacht werden müssten. ${ }^{92}$ Die Mehrzahl der Mediziner vertrat die Auffassung, dass die meisten Kinder unabhängig von ihrem sozialen Status gesund geboren wurden und dass die Verschlechterung ihres Zustandes das Resultat der verarmten Umgebung sei, in der sie aufwüchsen. ${ }^{93}$ Daneben wurde zu bedenken gegeben, dass die Armeebewerber keine repräsentative Gruppe der Gesamtbevölkerung darstelle, da die gesunden und fähigen Männer durch die lebhafte Kriegskonjunktur Arbeitsmöglichkeiten im eigenen Land fänden und deshalb nicht den Kriegsdienst wählten. Die Empfehlungen des Komitees sahen dann u.a. den weiteren Ausbau des öffentlichen Gesundheitswesens vor und verstärkte staatliche Inspektionen in Fabriken und Schulen. ${ }^{94}$ Wo Präventivmediziner und Verwaltungsangestellte als Gutachter die Oberhand behielten, wurde also vom Kurs eines starken environmentalism kaum abgewichen.

\subsection{Die Eugenics Education Society}

1907, im gleichen Jahr, in dem die Royal Commission on the Care and Control of the Feeble-Minded ihre Anhörungen abschloss, wurde die Eugenics Education

90 Siehe DavIE, Tracing the Criminal, S.247; zur Manchester Kalkulation (1899) siehe SolOwAY, Demography, S. 2, S. 41-43.

91 Report of the Inter-Departmental Committee on Physical Deterioration (1904). Bd. 1 (Report and Appendix), Parliamentary Papers (1905), Cd. 2175, S. 1.

92 Siehe SolowaY, Demography, S. 45.

93 Vgl. dazu ebd., S. 148.

94 Siehe Report Physical Deterioration, Summary of Recommendations, S. 84-93; zum InterDepartmental Committee siehe auch SolowaY, Demography, S. 43-47. 
Society gegründet. Galton, der an diesem Vorhaben nicht beteiligt war, versuchte sich mit ihrer Existenz zu arrangieren, indem er zwei Jahre später auf die Arbeitsteilung im Prozess der Wissensproduktion und ihrer Popularisierung hinwies: „There are two sorts of workers in every department of knowledge - those who establish a firm foundation, and those who build upon the foundation. "95 Während sich das durch Galtons finanzielle Unterstützung 1905 ins Leben gerufene Galton Laboratory am University College in London ${ }^{96}$ um die Etablierung von Eugenik als Wissenschaft durch biometrische Grundlagenforschung bemühte, ${ }^{97}$ stand für die Eugenics Education Society die Propagierung eugenischen Gedankenguts durch Aufklärung der Öffentlichkeit über die Mechanismen der Vererbung im Vordergrund. Man erklärte Galtons Definition von Eugenik zum Leitsatz der Gesellschaft: „Eugenics is the study of agencies under social control that may improve or impair the racial qualities of future generations either physically or mentally. "98

Galton selbst versuchte in seinem Vorwort zur ersten Ausgabe der ab 1909 herausgegebenen Eugenics Review die Aufgabe des Journals dahingehend zu bestimmen, „to give expression to the Eugenic movement and to place Eugenic thought, where possible, on a strictly scientific basis“. ${ }^{99}$ Mit diesem Satz hatte Galton eine Art Notbremse gezogen, denn im Grunde blieb er, obgleich ehrgeizig genug, eugenisches Wissen möglichst rasch und umfassend an die Öffentlichkeit zu bringen und daraus eine Art säkularer ,Religion' 100 zu machen, skeptisch gegenüber einer Gesellschaft, die seiner Auffassung nach zu wenig wissenschaftlich und zu stark politisch orientiert war. Wissenschaft, wie Galton sie verstand, gewährleistete eigentlich nur das Eugenics Laboratory, das seit 1907 von Karl Pearson als Direktor geleitet wurde. ${ }^{101}$ Galton sah durchaus, dass eine nach seiner Ansicht

95 Francis Galton, Foreword, in: The Eugenics Review 1 (1909), S. 1.

96 Das Eugenics Record Office wurde mit Galtons finanzieller Unterstützung 1905 ins Leben gerufen. Es war von Pearsons Biometrischem Labor getrennt. 1906 wurde aus dem Eugenics Record Office das Galton Laboratory mit Pearson als Direktor, siehe JONES, Theoretical Foundations, S. 17, Anm. 1.

97 Der genaue Name lautete Francis Galton Laboratory for the Study of National Eugenics; 1963 wurde es umbenannt in Galton Laboratory of the Department of Human Genetics and Biometry, siehe GILLHAM, Francis Galton, S. 334; zur Arbeit des Labors siehe das Ed7itorial von Karl Pearson in der ersten Ausgabe von Biometrika. A Journal for the Statistical Study of Biological Problems 1 (1901-1902), S. 3: „Whatever views we hold on selection, inheritance, or fertility, we must ultimately turn to mathematics of large numbers, to the theories of mass-phenomena, to interpret safely our observations." Vgl. dazu auch den Artikel von FRANCIS GALTON, Biometrika, in: Biometrika 1 (1901-1902), S.7-10.

98 Dieses Motto wurde in jeder Ausgabe der Eugenics Review abgedruckt; vgl. dazu auch Galtons Definition in seinem Vortrag auf der Gründungveranstaltung der Sociological Society 1904: „Eugenics is the science which deals with all influences that improve the inborn qualities of a race; also with those that develop them to the utmost advantage." GaLTON, Eugenics: Its Definition, S. 45.

99 Galton, Foreword, S. 1.

100 Vgl. dazu Francis GaLton, in: Nature (1901), S. 663f., wo er Eugenik als eine Aufgabe bezeichnet, die so noble sei, dass sie Anlass zum Gefühl einer neuen religiösen Verpflichtung gebe.

101 Siehe dazu Kap. 5 dieser Arbeit; zu Pearsons Arbeit am University College siehe M. EILEEN MagnelLo, The Non-Correlation of Biometrics and Eugenics: Rival Forms of Laboratory 
verfrühte Popularisierung der Eugenik nicht nur die Gefahr einer wissenschaftlichen Verflachung mit sich bringen, sondern auch einer politischen Instrumentalisierung dienen könnte, die die von ihm behauptete Wissenschaftlichkeit in erster Linie zur Legitimation sozialpolitischer Forderungen einsetzen würde. Bereits 1904 hatte er in einem Vortrag anlässlich der Gründung der Sociological Society vor einer vorschnellen Umsetzung und falschen Hoffnung gewarnt:

I see no impossibility in Eugenics becoming a religious dogma among mankind, but its details must first be worked out sedulously in the study. Over-zeal leading to hasty action would do harm, by holding out expectations of a near golden age, which will certainly be falsified and cause the science to be discredited. The first and main point is to secure the general acceptance of Eugenics as a hopeful and most important study. ${ }^{102}$

1908 nahm die Eugenics Education Society ihre Tätigkeit auf und formulierte ihre Aufgaben und Ziele dementsprechend: „Persistently to set forth the National Importance of Eugenics in order to modify public opinion, and create a sense of responsibility in the respect of bringing all matters pertaining to human parenthood under the domination of Eugenic ideals. "103 Die Eugenische Gesellschaft verstand sich als gemeinnütziger Verein, der sich von anderen sozialreformerischen Gesellschaften lediglich in der Wahl der Mittel zur Verbesserung der sozialen Schwachstellen der Nation unterschied. Sie war allerdings die erste und einzige, die nicht mehr den Weg sozialer Verbesserungen durch aktive Eingriffe in das soziale Umfeld der Betroffenen einschlug, sondern ausschließlich auf biologische Erklärungen und die Umsetzung biologischer Konzepte setzte. Zum Eugenics Laboratory am University College wollte die Eugenische Gesellschaft ausdrücklich nicht in Konkurrenz treten. Das auf mathematische und statistische Berechnungen konzentrierte Unternehmen lanciere doch, so ließ man vernehmen, mehr ,technische' Publikationen. Karl Pearson vom Labor sah die Sache dagegen ganz anders. Er hegte große Sorge, dass seine wissenschaftliche Arbeit mit einer populistischen Vereinigung in Verbindung gebracht wurde. ${ }^{104}$

Aus welchen Kreisen stammten die Mitarbeiter der Eugenics Education Society, die sich als gemeinnützige Organisation begriff? Wie Donald MacKenzie gezeigt

Work in Karl Pearsons's Career at University College, London, in: History of Science 37 (1999), S. 79-106, S. 123-150.

102 Francis Galton, Eugenics: Its Definition, Scope and Aims, S. 50, Hervorhebung S.F.; 1909 akzeptierte Galton die Ehrenpräsidentschaft der EES.

103 Vgl. die „Objects of the Eugenics Education Society“, abgedruckt auf der Umschlaginnenseite einer jeden Ausgabe der Eugenics Review. Die beiden weiteren Punkte lauteten: „2. To spread the knowledge of the Laws of Heredity so far as they are surely known, and so far as that knowledge may affect the improvement of the race. 3. To further Eugenic Teaching at home, in the schools, and elsewhere." Vgl. dazu auch SolowaY, Demography, S.32; DaVIE, Tracing the Criminal, S.248.

104 Zum Streit zwischen der EES und dem Labor siehe Gillham, Francis Galton, S. 338-342; zu Pearsons Ablehnung ebd. S. 339; BrooKes, Extreme Measures, S. 285-291; STEFAn KüHL, Die Internationale der Rassisten. Aufstieg und Niedergang der internationalen Bewegung für Eugenik und Rassenhygiene im 20. Jahrhundert, Frankfurt am Main 1997, S.245, Anm. 31. 
hat, waren es vor allem Mitglieder einer professional middle class. ${ }^{105}$ Die meisten Vereinsmitglieder übten Berufe aus, die ein hohes Maß an intellektuellen Fähigkeiten voraussetzten, z. B. technische Berufe. Diese brain workers ${ }^{106}$ hatten die Chancen eines sich öffnenden Bildungssystems zu ihrem Aufstieg nutzen können und betrachteten sich selbst als die eigentlichen Leistungsträger der englischen Gesellschaft. Die meisten Mitglieder - darunter viele Frauen - ${ }^{107}$ hatten keinen Anteil am großen Kapital, wie es die alte Geburtsaristokratie immer noch und die Schicht bürgerlicher Industrieller neuerdings besaß. Die eigene finanzielle Situation gab zwar in der Regel keinen Anlass zur Klage, war aber auch nie solcherart gesichert, dass man gelassen in die Zukunft hätte blicken können. Die Sorge blieb bestehen, ob auch die eigenen Nachkommen den gleichen professionellen Status erlangen und halten konnten. Das Übergewicht an Mitgliedern aus der professionellen und akademischen Mittelschicht verursachte aufschlussreiche Leerstellen in der Mitgliederstruktur der Gesellschaft. Verglichen mit anderen Reformgesellschaften fehlten Politiker in der EES fast ganz, ebenso wie Unternehmer und Geschäftsleute ${ }^{108}$ sowie - wenig überraschend - Mitglieder aus den Gewerkschaften. Aus leicht einsehbaren Gründen gab es auch keine Mitglieder aus den sogenannten „environmental professions: local government officials, civil servants and social workers“.109

In der Rolle einer Vermittlerin zwischen Wissenschaft und Öffentlichkeit war die Eugenics Education Society kein Ort exklusiver Wissensproduktion, sie war

105 Vgl. Donald MacKenzie, Statistics in Britain, 1865-1930: The Social Construction of Scientific Knowledge, Edinburgh 1981, S. 24-28; zu den Mitgliedern der Gesellschaft siehe auch Lyndal A. FarRall, The Origins and Growth of the English Eugenics Movement, 1865-1925, New York 1985 (zugleich University of Indiana Diss. 1969), S. 210-230; Geoffrey R. SEARLE, Eugenics and Politics in Britain, 1900-1914, Leyden 1976, S. 10-14; ders. Eugenics and Class, in: Webster (Hrsg.), Biology, Medicine and Society, S. 217-241, hier: S. 225-227; IAN BrowN, Who Were the Eugenicists? A Study of the Foundation of an Early Twenthieth Century Pressure Group, in: History of Education 17 (1988), S. 295-307. Die Mitgliederzahl vor dem Ersten Weltkrieg wird auf 600-1000 geschätzt, siehe die Zahlen bei RADZINOwICZ und HooD, The Emergence of Penal Policy, S.31; Sim, Medical Power, S. 141.

106 Vgl. dazu Pearsons Definition der neuen Mittelklasse, die selbst aus Selektion hervorgegangen sei: „[T]he middle class in England, which stands there for intellectual culture and brain-work, is the product of generations of selection from other classes and of inter-marriage." Karl Pearson, The Function of Science in the Modern State (1902), Cambridge 1919 [Reprint], S.9f.

107 Zum Verhältnis von Frauen und Eugenik siehe Richard SolowaY, Feminism, Fertility, and Eugenics in Victorian and Edwardian England, in: Seymour Drescher, David Sabean und Allan Sharlin (Hrsg.), Political Symbolism in Modern Europe, New Brunswick 1982, S. 121145; GRETA JONES, Women and Eugenics in Britain: the Case of Mary Scharlieb, Elizabeth Sloan Chesser, and Stella Browne, in: Annals of Science 51 (1995), S. 481-502; LuCY BLAND, Banishing the Beast: English Feminism and Sexual Morality, 1885-1914, London 1995; LesLEY A. HALL, Women, Feminism and Eugenics, in: The Galton Institute (Hrsg.), Essays in the History of Eugenics, London 1998, S. 36-49.

108 Dagegen hat GRETA JONES (Social Hygiene in Twentieth Century Britain, London 1986, S. 1921) allerdings eingewendet, dass in den Eugenischen Gesellschaften von Birmingham, Manchester und Liverpool, und in den 1930er Jahren auch in der Londoner EES, eine Reihe von Geschäftsleuten Mitglieder gewesen seien; zur Diskussion über die Mitglieder-Zusammensetzung siehe SEArLE, Eugenics: The Early Years, S. 30-33.

109 Searle, Eugenics and Class, S. 235. 
aber ebenso wenig ein Ort, an dem Wissenschaft ausschließlich popularisiert und verbreitet wurde. Ihre Mitglieder betrieben eigenständige Forschung, in dem sie Galtons frühe Stammbaumanalysen aufgriffen und weiterentwickelten. Sie wählten damit ein Verfahren, dass ähnlich wie die von der Statistical Society betriebene Sozialstatistik vor allem eine hohe Zahl an Mitarbeitern benötigte, die Familieninterviews durchführten und medizinische Akten auswerteten. Eine überdurchschnittliche mathematische Begabung, wie sie für die Mitarbeiter des biometrischen Labors notwendige Voraussetzung war, war nicht notwendig. Die Forschungstätigkeit bzw. -leistung bestand im massenhaften Sammeln von Daten und Informationen von Familienverbänden unterschiedlichster Provenienz und ihre graphische Umsetzung in Stammbäume. Die so erstellten Stammbaumtafeln sollten in direkter und anschaulicher Weise dem Betrachter die zentrale eugenische Grundannahme vermitteln: Bestimmte Krankheiten, aber auch Charaktereigenschaften wurden von den Eltern an die Nachkommen weitergeben. ${ }^{110}$ Dass biologische Prozesse, Anlage und nicht Umwelt, also nature und nicht nurture, wie Galton es bereits 1873 prägnant auf eine Formel gebracht hatte, ${ }^{111}$ das menschliche Leben im Wesentlichen bestimmten, diese Wahrheit sollte sich dem Betrachter der graphisch aufbereiteten pedigrees als unmittelbare Evidenz aufdrängen. ${ }^{12}$ Die Notwendigkeit eines aktiven Eingreifens in den Reproduktionsprozess betroffener Familien sollte sich dadurch erschließen. Die Stammbäume waren, wie die Medizinhistorikerin Pauline Mazumdar schreibt, für die meisten Mitglieder tatsächlich „a straightforward demonstration that like engendered like, with no specific theory of inheritance implied." 113

Tatsächlich nahm die Eugenics Education Society, besonders vor dem Ersten Weltkrieg, eine verblüffend unkritische Haltung sowohl gegenüber den eigenen Forschungsmethoden als auch den konkurrierenden wissenschaftlichen Vererbungstheorien ein. Dass die Befragung von Familienangehörigen über andere Familienangehörige, die man womöglich nicht einmal persönlich gekannt hatte, zu Ungenauigkeiten und Fehlern führen konnte, etwa in der Einschätzung von Alkoholismus, wurde nicht thematisiert. Auch an der theoretischen Kontroverse zwischen den Biometrikern und den Anhängern der Mendelschen Vererbungs-

$110 \mathrm{Zu}$ diesem Verfahren hatte Francis Galton selbst in seinem Buch Hereditary Genius (1869) die Vorlage geliefert.

111 Francis Galton hat nicht nur den Begriff Eugenik nach dem griechischen ev́yéveı (edle Abkunft, Adel) geprägt (vgl. Francis GALTON, Inquiries into Human Faculties and Its Development, London 1883, S.24f.), sondern auch die prägnante Kurzformel nature/nurture für den Gegensatz von Anlage und Umwelt geschaffen (vgl. Francis GalTon, English Men of Science: Their Nature and Nurture, London 1873); siehe dazu auch RuTH SCHWARZ CowAN, Nature and Nurture: The Interplay of Biology and Politics in the Work of Francis Galton, in: Studies in the History of Biology 1 (1977), S. 133-208.

112 Zum Appell an den common sense siehe aufschlussreich EDWARD J. LARSON, The Rhetoric of Eugenics: Expert Authority and the Mental Deficiency Bill, in: British Journal for the History of Science 24 (1991), S. 45-60.

113 Pauline M.H. Mazumdar, Eugenics, Human Genetics and Human Failings. The Eugenics Society, its sources and its critics in Britain, London und New York 1992, S. 4. 
lehre nahm die EES nicht aktiv teil. ${ }^{114}$ Beide Forschergruppen betrachtete man als Helfer für die eugenische und damit, aus Sicht der Mitglieder, gemeinnützige Sache. Die pragmatische Empfehlung des ersten Präsidenten der Gesellschaft, Montague Crackanthorpe, lautete deshalb auch: „Eugenics requires the services of both." 115 Vertretern beider Schulen stand die Eugenics Review als Publikationsorgan zur Verfügung. Zur Präsentation ihrer jeweiligen Forschungen konnten auch die Veranstaltungen der Gesellschaft genutzt werden. Von den vielen offenen Fragen beider Wissenschaftsrichtungen ließen sich die meisten Mitglieder nicht verunsichern. Bedenken meldeten nur, so wird zu zeigen sein, jene Mitglieder an, die sich selbst zum Kreis der Wissenschaftler zählten.

\subsection{Eugenik und Kriminalität}

Obwohl sich die EES beim Sammeln von Daten und Informationen vorzugsweise auf ,degenerierte' Familien konzentrierte, also auf Familien, die von Armut, Krankheit und Kriminalität gekennzeichnet waren, nahmen die Themen Kriminalität und Kriminelle einen sehr bescheidenen Raum innerhalb der Gesellschaft ein. Die Erklärung des Phänomens Kriminalität war für die meisten Eugeniker wenig problematisch: Chronisch Arbeitslose, Arme, Kranke und Kriminelle waren alle Opfer ihrer Natur, die Ursache ihres Zustands ließ sich auf eine körperliche und/oder geistige Minderwertigkeit zurückführen. Der Versuch von Besserung oder Reformierung durch Veränderung des sozialen Umfeldes schien hier fehl am Platze, nur systematisches Herauszüchten solcher Erbanlagen durch ein Fortpflanzungsverbot von Trägern negativer Anlagen, bei gleichzeitiger Stimulation der Reproduktion von Trägern positiver Anlagen, konnte nach Überzeugung der Eugeniker langfristig greifen. Während die Sozialbehörden die sozial schwächeren oder abhängigen Mitglieder der britischen Gesellschaft in administrativer Sprache einfach als diejenigen Personen bezeichneten, die auf öffentliche Unterstützung angewiesen waren, sahen Eugeniker den gleichen Personenkreis als Träger real existierender, durch Ärzte diagnostizierbarer Defekte, die sie von den civic oder social worth klar unterschied. Unter britischen Eugenikern war es eine Selbstverständlichkeit, Kriminelle im gleichen Atemzug mit den undeserving poor, ${ }^{116}$

114 Zur dieser Kontroverse siehe Kap.5.3.

115 Montague Crackanthorpe, Presidential Address, 5 May 1910, Eugenics Education Society, Second Annual Report (1909-10), S. 1-16, hier S. 5.

116 Dieser Begriff hatte sich bei zahlreichen Wohltätigkeitsvereinen und in der Presse eingebürgert, um jene Wohltätigkeitempfänger zu bezeichnen, die auf Hilfe nicht entsprechend reagierten, sich also resistent zeigten und deshalb von der Unterstützung ausgeschlossen werden sollten, vgl. dazu z. B. Gertrude Himmelfarb, Poverty and Compassion: The Moral Imagination of the Late Victorians, New York 1991, S. 12; JANE LEWIS, Woman and Social Action in Victorian and Edwardian England, Stanford 1991, S. 15; dies, Woman and Late-Nineteenth-Century Social Work, in: Carol Smart (Hrsg.), Regulating Womanhood. Historical Essays on Marriage, Motherhood and Sexuality, London und New York 1992, S. 78-99, hier S. 81; RICHARDSON, Love and Eugenics, S. 17. 
den Geisteskranken, den unsteten Vagabunden, den Tuberkulosekranken und den Epileptikern zu nennen. ${ }^{117}$ Kriminelle zählten damit zu jener Trägergruppe, die in ihrer Gesamtheit die für eine gesunde Gesellschaft nicht wünschenswerten Erbanlagen repräsentierte. Ein genaueres Nachdenken über die Voraussetzungen dieser Annahme schien nicht besonders dringlich. Jedenfalls erstaunt bei einer Durchsicht der Eugenics Review für die Zeit von 1909 bis 1939 die geringe Zahl an Artikeln zum Thema Kriminalität. Wurde Kriminalität überhaupt zum Thema gemacht, dann nur von einigen wenigen Eugenikern.

Einen programmatischen Beitrag zum Thema Kriminalität und Eugenik lieferte 1914 Leonard Darwin, der zweitjüngste von Charles Darwins fünf Söhnen. ${ }^{118}$ Als Präsident der Gesellschaft von 1911 bis 1929 widmete er sich in seiner Jahresansprache dem seit der Jahrhundertwende in der Öffentlichkeit verstärkt diskutierten Problem des Gewohnheitsverbrechers. ${ }^{119}$ Selbst kein Naturwissenschaftler oder Mediziner, sondern wie Edmund Du Cane Absolvent einer militärischen Ausbildung bei den Royal Engineers, hatte Leonard Darwin die letzten fünf Jahre seines Berufslebens als Mitglied des Intelligence Department im Kriegsministerium verbracht, bevor er 1890 aus dem aktiven Dienst ausschied. Darwin interessierte sich für ökonomische Fragen und kam über seine Beschäftigung mit der national efficiency zur Eugenik. ${ }^{120}$ Wie Galton trat er dabei vor allem als Befürworter einer positiven Eugenik auf, „the judicious mating of mankind“, 121 wie er es in seinem Vortrag nannte. Nur in Zusammenhang mit Kriminellen und Geisteskranken spielte negative Eugenik, also ein Reproduktionsverbot durch Segregation oder Sterilisation, bei ihm eine prominente Rolle. Bereits 1912 hatte Leonard Darwin ein Nationalregister der „naturally unfit“ gefordert, in das er alle „insand-outs at work-houses, and all convicted prisoners"122 verzeichnet sehen wollte. Durch ein solches System erhoffte er sich gründlichere Stammbaumanalysen von Familien, „especially as regards the criminality, insanity, ill health and pauperism of their relatives; thus many traits would be discovered which no one could deny ought to be made to die out in the interest of the nation". 1231927 arbeitete Darwin einen Gesetzentwurf zur freiwilligen Sterilisation aus. ${ }^{124}$

Für Leonard Darwin stand außer Frage, dass es einen Zusammenhang zwischen Kriminalität und biologischer Vererbung gab. Die seit dem Ende des

117 Vgl. Searle, Eugenics and Politics, S. 62.

118 Major Leonard Darwin, The Habitual Criminal. Presidential Address Delivered at the Annual General Meeting of the Eugenics Education Society, July 2 ${ }^{\text {nd }}, 1914$, in: Eugenics Review 6 (1914), S.204-218.

$119 \mathrm{Zu}$ Diskussion über den Gewohnheitsverbrecher siehe RadzINOwICZ und Hood, Emergence of Penal Policy, S. 231-287; Freitag, A Perverse Determination, S. 219-229.

120 Zur Diskussion über die national efficiency siehe bes. SolowAY, Counting the Degenerates, S. 137-164; Robert A. Lowe, Eugenicists, Doctors and the Quest for National Efficiency: an Educational Crusade, 1900-1939, in: History of Education 8 (4/1979), S. 293-306.

121 Darwin, Habitual Criminal, S. 218.

122 Vgl. Leonard Darwin, First Steps Towards Eugenic Reform, in: Eugenics Review 4 (1912), S. 34-35.

123 Ebd.

124 Vgl. dazu Davie, Tracing the Criminal, S. 254. 
19. Jahrhundert vieldiskutierte Erscheinung der Rückfälligkeit (recidivism) deutete er nicht in erster Linie als Produkt verbesserter Statistiken und vernetzter Institutionen wie Polizei, Gericht und Gefängnis, sondern als Indiz für eine angeborene Minderwertigkeit. Gerade der Typus des Gewohnheitsverbrechers, der durch die ununterbrochene Wiederholung seiner Straftaten und seine Resistenz gegenüber Strafe und Reformierung auffiel, verwies auf die Existenz pathologischer Persönlichkeitsstrukturen. Zwar war auch Darwin bereit zuzugeben, dass es sich bei den kriminellen Tendenzen dieser Gewohnheitsverbrecher nicht um eine „single heritable quality“, 125 sondern höchstwahrscheinlich um das Ergebnis eines Zusammenspiel zahlreicher verschiedener „innate qualities“126 handelte, aber an ihrer erblichen Übertragung hielt er dennoch fest. Direkte Beweise dafür konnte er nicht liefern.

Wie für die meisten Eugeniker standen auch bei Darwin Anwendungsfragen im Vordergrund. Wie sollte man mit dem „man of many petty crimes“127 verfahren, der doch durch sein wiederholtes anti-soziales Verhalten die Gesellschaft am nachhaltigsten schädigte? Da es sich nicht lohne, dessen Qualitäten unter hohen Kosten weiterhin zu tolerieren, müsse die erste Aufgabe sein, „to pick those who are endowed to a very exceptional extent with natural qualities which facilitate the adoption of a life of crime." 128 Sei dies bewerkstelligt, dann sei das probateste Mittel die „segregation of these criminals during the period of their fertility [...] to prevent these innate qualities from reappearing in future generations." 129 Zur Identifizierung von Gewohnheitsverbrechern empfahl Darwin die Zahl der begangenen Straftaten als ein erstes untrügliches Zeichen in den Blick zu nehmen. ${ }^{130}$ Aufschlussreich konnte darüber hinaus auch das Alter des Straftäters sein, in welchem er seine kriminelle Karriere begonnen hatte, denn je stärker die natürliche Anlage zu kriminellen Handlungen ausgeprägt sei, desto früher, so glaubte Darwin, verschaffe sie sich Geltung. Schließlich gab es noch die ganz offensichtlichen körperlichen und geistigen Gebrechen, die bereits in anderer, vor allem medizinischer und psychiatrischer Literatur erwähnt wurden. Anhand dieses Erkennungs-Kataloges sollte es nach Darwins Auffassung jedem „well-instructed official“131 möglich sein, ohne Schwierigkeiten die große Zahl von Individuen herauszufiltern, die man als Träger dieser für die Gesellschaft schädlichen Anlagen identifizieren könne.

Mit der Vorstellung, dass es erbliche Dispositionen gab, die eine Neigung zu Kriminalität wahrscheinlich machten, verbunden mit der Forderung eines strikten Fortpflanzungsverbots, stand Darwin nicht alleine. ${ }^{132}$ Den Zusammenhang

125 Darwin, Habitual Criminal, S. 207.

126 Ebd., S. 207.

127 Ebd., S. 209.

128 Ebd., S. 210.

129 Ebd., S. 211.

130 Ebd.

131 Ebd.

$132 \mathrm{Zu}$ weiteren Befürwortern von Zwangs- und freiwilligen Sterilisationen von Straftätern siehe DAVIE, Tracing the Criminal, S.253-259, zu den Befürwortern von Zwangssterilisationen zählten die Gefängnisärzte und Anstaltspsychiater Richard F. Quinton und Thomas Clous- 
von Kriminalität und Vererbung hatte schon Galton öffentlich postuliert. In seinen Inquiries into the Human Faculties (1883) hatte er kriminelles Verhalten auf bestimmte Charaktereigenschaften zurückgeführt, auf das Fehlen oder die gestörte Funktion bestimmter moralischer und/oder mentaler Fähigkeiten, die er nicht durch Umweltfaktoren bedingt glaubte, sondern für das Resultat biologischer Vererbung hielt: „[T]he ideal criminal has marked peculiarities of character, his instincts are vicious, his power of self-control is very weak and he usually detests continuous labour. The absence of self-control is due to ungovernable temper, to passion or to mere imbecility [...] it is easy to show that the criminal nature tends to be inherited."133 1901 hatte Galton dann in einem Vortrag über The Possible Improvement of the Human Breed Under the Existing Conditions of Law and Settlement die Konsequenzen formuliert, die eine solche Erkenntnis erforderte. Mit Bezug auf die Großstudie von Charles Booth aus den 1890er Jahren griff Galton dessen Idee von Arbeitskolonien auf. ${ }^{134}$ Da es den Anschein habe, dass die Eigenschaften der ,Klasse A', die Booth als arbeitsscheu, unstet, halb-kriminell und kriminell beschrieben hatte, ${ }^{135}$ in einem beachtlichen Ausmaße als erblich angenommen werden müssten, wäre es eine große Entlastung, „if all habitual criminals were resolutely segregated under merciful surveillance and peremptorily denied opportunities for producing offspring. "136 Während Galton diese Idee zwar aufführte, aber nie weiter verfolgte, sah der führende Eugeniker und Fabian socialist, Dr. Caleb Williams Saleeby, in ihr schon den zentralen Bestandteil eines „Scientific Patriotism“. Ohne Frage sei es „well worth society's while that the genius and the saint, the athlete and the artist, should provide posterity, rather than the idiot, the criminal, [and, S.F.] the weakling ". ${ }^{337}$

Obwohl Leonard Darwin die Ergebnisse von Charles Gorings The English Convict hätte aufgreifen können, der zufolge sich Gewohnheitsverbrecher durch defekte Intelligenz auszeichneten und als potentielle Eltern ein zukünftiges Element

ton, Scotland Yard-Beamte wie Sir Robert Anderson, Mediziner wie Dr. Robert Reid Rentoul; zu den Befürwortern einer freiwilligen Sterilisation zählten Havelock Ellis und Alfred Tredgold; insgesamt bildete diese Gruppe aber eine Minorität, siehe dazu SolowaY, Demography, S. 64; Searle, Eugenics: The Early Years, S. 33; DAvIE, Tracing the Criminal, S. 256: „But - unlike in US: they never succeeded in making significant inroads into political, administrative or medical elite opinion."

133 Francis Galton, Inquiries into Human Faculty and its Development, London 21883, S. 4243; hier zit. nach DownING und FonsYTHE, The Reform of Offenders in England, S. 150.

134 Charles Booth hatte Arbeitskolonien auf dem Land empfohlen, wo „their half-fed and halfidle and wholly unregulated life“ ausgetauscht werden würde gegen eine „disciplined existence, with regular meals and fixed hours of work (which would not be short)." Hier zit. nach Stedman-Jones, Outcast, S. 307; auch DaVIE, Tracing the Criminal, S. 252.

135 Vgl. Kap. 2.6.

136 Francis Galton, The Possible Improvement of the Human Breed Under the Existing Conditions of Law and Settlement, in: Nature, 31. Oktober 1901, S. 659-665, hier S. 663 (Vortrag im Anthropologischen Institut London).

137 Beide Zitate: Caleb W. Saleeby, Parenthood and Race Culture: An Outline of Eugenics, New York 1910, S. 30, hier zit. nach DAVIE, Tracing the Criminal, S.253; Saleeby überwarf sich später mit der EES, der er vorwarf, sie werde immer mehr zu einer Bewegung, die sich durch reaktionäre Klassenvorurteile auszeichne, siehe SEARLE, Eugenics: The Early Years, S. $21 \mathrm{f}$. 
nationaler ,Degeneration' darstellten, ${ }^{138}$ mußte er in seinem Vortrag von 1914 dennoch zuzugeben, dass die öffentliche Meinung eine unbestimmte Haft oder Sicherungsverwahrung für diese Straftäter besonders während ihrer fruchtbaren Jahre nicht tolerieren würde. ${ }^{139}$ „Unfortunately“, so resümierte Darwin in seinem Vortrag, „such broad considerations weigh but little with the general public. We shall therefore be wise in regarding this question of natural heritability as at present a weak part of our armour when attacking problems connected with criminality. " 140 So erfolgreich sich Vererbungslehre und -theorie auch entwickelten, eugenische Maßnahmen für Kriminelle wurden damit noch lange nicht selbstverständlich: „[T] o convince the public of the advisability of prolonged segregation in his [the habitual criminal's, S.F.] case on eugenic grounds is a very different matter from convincing the student of heredity; and it must be admitted that our proofs of the heritability [...] are not now sufficiently convincing to enable us to found on them a bold eugenic policy. "141 Die gleichen Bedenken formulierte Darwin noch einmal in seinem 1926 publizierten, dem Vater gewidmeten Buch über The Need for Eugenic Reform. ${ }^{142}$ Das Buch war in seiner nun formulierten Zurückhaltung und Vorsicht bereits ein Ausdruck für die Reaktion Darwins auf die öffentliche Kritik, die das eugenische Reformprogramm seit der Gründung der EES von unterschiedlichen Seiten erfahren hatte.

\subsection{Zur wissenschaftlichen Kritik eines sozialpolitischen Programms}

Allgemein bekannt ist - wenn auch noch nicht zum Gegenstand einer systematischen Untersuchung geworden -, dass der Eugenics Education Society in Großbritannien eine engagierte öffentliche Kritik entgegenschlug, die besonders von solchen Kreisen getragen wurde, die sich durch die offensichtliche Klassenideologie der Eugeniker direkt angegriffen fühlten. ${ }^{143}$ Die Labour Party und die Ge-

138 Dazu ausführlicher Kap. 5.

139 Vgl. dazu auch LeONARd Darwins Bemerkungen auf einem Treffen der EES, auf dem er seiner Hoffnung Ausdruck gab, dass psychologische Test entwickelt werden könnten, mit deren Hilfe zwischen solchen Kriminellen, die mit Sicherheit entlassen werden könnten, und solchen, die permanent in Gewahrsam genommen werden müssten, unterschieden werden könne; abgedruckt in: British Medical Journal (1913), S. 1321; der Hinweis findet sich bei Radzinowicz und Hood, The Emergence of Penal Policy, S. 33.

140 Darwin, Habitual Criminal, S. 208.

141 Ebd., S. 212.

142 Leonard Darwin, The Need for Eugenic Reform, London 1926, bes. Kap. 13: The Habitual Criminal, S. 206-226; die aufschlussreiche Widmung des Buches lautet: „Dedicated to the Memory of My Father For if I had not believed that He would have wished Me to give such Help as I Could towards Making His Life's Work of Service to Mankind, I should never have been led to write this Book.“

143 Diese Klassenideologie hat die englische Eugenik von der deutschen oder amerikanischen Rassenideologie wesentlich unterschieden; Stefan Kühls internationale Organisationsgeschichte der Eugenik (siehe KüHL, Internationale) leidet darunter, dass sie auf eine differen- 
werkschaften als Interessenvertreter der Arbeiterschichten zählten dazu ebenso wie Mitglieder der katholischen Kirche, die Ehe, Familie und Kinderzeugung ihrer Zuständigkeit zurechneten. Zu den bekanntesten katholischen Kritikern gehörte Gilbert Keith Chesterton, der in seinem 1922 publizierten Buch Eugenics and Other Evils mit guten Gründen gegen den „scientifically organised state“ anschrieb. ${ }^{144}$

Im Kontext dieses Kapitels interessiert zunächst, welche Kritik die Eugenik aus der aufgrund der englischen Wissenschaftskultur recht weit gefassten scientific community erfahren hat, denn diese Kritik beeinflusste auch den Diskurs über Kriminalitätsgenese und Verbrecherkonstitution. Wenig bekannt ist, dass die wissenschaftliche Kritik an der Eugenik nicht nur von solchen Ärzten, Biologen und Sozialreformern vorgetragen wurde, die der Gesellschaft ohnehin fern standen, sondern dass sich auch innerhalb der Gesellschaft einige Mitglieder recht früh kritisch mit den wissenschaftlichen Voraussetzungen ihres sozialpolitischen Programms auseinander setzten. Diese Einwände wurden bereits vor dem Ersten Weltkrieg formuliert.

\section{"The production of better citizens". Zur wissenschaftlichen Kritik von außen}

Kritik am eugenischen Reformprogramm der EES kam nicht nur von außen, sondern auch aus den Reihen ihrer eigenen Mitglieder. Was die Kritik von Wissenschaftlern betrifft, die der EES nicht nahe standen, lässt sich hier zunächst an die Ergebnisse des 3. Kapitels über die Präventivmedizin und -psychiatrie anknüpfen. Die Einwände, die gegen Galtons Vortrag über Eugenics: Its Definition, Scope and Aims auf der Gründungsveranstaltung der Sociological Society 1904 an der London School of Economics vorgebracht wurden, machen dies deutlich. In der Diskussion, die sich an den Vortrag anschloss, meldeten sich sofort prominente Kritiker zu Wort, die Galtons Programm und seine Voraussetzungen für problematisch hielten. Henry Maudsley warnte vor falschen Analogien zwischen Mensch und Tier. Seine Erfahrung habe ihn gelehrt, dass Mitglieder ein- und derselben Familie unterschiedlicher nicht sein könnten: Während ein Mitglied zu höchsten Ehren aufsteige, leide ein anderes Mitglied an einer schweren geistigen Störung. Als prominentes Beispiel verwies er auf William Shakespeare. Er sei von Eltern geborenen worden, die sich nicht von ihren eigenen Eltern unterschieden hätten

zierte Darstellung der nationalen Eugenik-Vorstellungen verzichtet. Dadurch kann sie die Kontroversen und Diskrepanzen zwischen den einzelnen Bewegungen, unter denen ja dann auch die Zusammenarbeit litt, nicht wirklich deutlich machen, denn mit der „Aufartung der Rasse" (ebd. S. 15) und der Förderung von civic worth waren ganz andere Strategien und Ziele verbunden; zu Kühls Programm einer internationalen Organisationsgeschichte ohne differenzierte Berücksichtigung der nationalen Rahmenbedingungen und Ideologien, vgl. KüHL, Internationale, S. 242, Anm. 6.

$144 \mathrm{Zu}$ Chestertons Kritik siehe ausführlicher Kap.5.9.; auch FreITAG, We Cannot Wait, S. 302306. 
und von seinen fünf Brüdern „none distinguished themselves in any way."145 Maudsley gab zu bedenken:

$[\mathrm{W}]$ hen we come to the human being there are complexities which arise from the mental state - its moods and passions - which entirely disturb any conclusions which we are able to form from our observation of the comparatively simple machines which animals are. In view of these difficulties of the subject I think that we must not be hasty in coming to conclusions and laying down any rules for the breeding of human beings [...] I am not sure but that Nature in its own blind impulsive way does not manage things better than we can by any light of reason or by any rules which we can at present lay down. ${ }^{146}$

Auch Charles Mercier erinnerte an die Komplexität der Vererbungsmechanismen und warnte vor voreiligen Schlüssen, die dann auch noch eine praktische Umsetzung erfahren sollten. Er thematisierte das zentrale Problem von individueller Verantwortung und Zurechnung und führte die Vorstellung einer elterlichen Haftung für die Verbrechen ihrer erwachsenen Kinder aufgrund der elterlichen Weitergabe von körperlichen und geistigen Vermögen an ihre Kinder ad absurdum:

For, in so far as we know these laws, they are so obscure and complex that to us they work out as chance. [...] It is quite impossible to predict from the constitution of the parents what the constitution of the offspring is going to be, even in the remotest degree $[\ldots]$ and we cannot go back from the offspring and say what the parentage was. If we follow the customs of the Chinese and ennoble the parents for the achievements of their children, ${ }^{147}$ are we to hang the parents when the offspring commit murder? [...] What are suitable and unsuitable marriages? How are we to decide? ${ }^{148}$

Für die Aufnahme und Stellung der Eugenik in England war es entscheidend, dass es eine Gruppe von Sozialreformern, Hygienemedizinern und civil service Angehörigen gab, die bereits an der Entwicklung eigener Programme zur Bekämpfung sozialer Probleme mit ihren je eigenen Mitteln arbeitete. Die Konfrontation mit Eugenikern wie Karl Pearson und seiner Kritik an der maudlin philanthropy provozierte in dieser Gruppe eine deutliche Abwehrhaltung gegen die Eugeniker. Dr. Robert Hutchinson, Arzt in einem Kinderkrankhaus, sah in eugenischen Maßnahmen einen völlig falschen Ansatz und darüber hinaus überhaupt keinen Grund, warum man „the raw material of the race“ verbessern zu müssen glaubte. Dieses Material sei nicht schlecht, sondern lediglich schlecht ernährt. Man täte weitaus besser daran, sich auf praktische Fragen und den „food factor“ zu konzentrieren, anstatt sich in einer Masse wissenschaftlicher Theorien über Vererbung zu verlieren, über die man zum gegenwärtigen Zeitpunkt wenig Aussagen treffen könne. ${ }^{149}$

Ein klarer und eindrucksvoller Beleg für die Haltung der Environmentalisten sind auch die Einwände des Medical Inspector to the Local Government Board of

\footnotetext{
145 Kommentar von Henry Maudsley in Sociological PaPers (1904), S. 54.

146 Ebd.

147 Galton hatte das Beispiel angeführt, dass in China die Eltern für die Leistungen ihrer Kinder gelobt würden, weil sie im biologischen Sinne, wesentlichen Anteil an ihrem Erfolg hätten, vgl. Galton, Eugenics: Its Definition, Scope and Aims, S. 49.

148 Charles Mercier in Sociological Papers (1904), S. 55.

149 Zitat und Aussagen von Robert Hutchisonin in Sociological PaPers (1904), S. 58.
} 
Scotland William Leslie MacKenzie, die er in seinem schriftlichen Kommentar zu Galtons Grundsatzpapier, das gemeinhin als Gründungsdokument der eugenischen Bewegung gilt, im Namen der Hygieniker verfasste. ${ }^{150}$ Aufschlussreich ist MacKenzies Kommentar für den Kontext der vorliegenden Arbeit deshalb, weil er sich darin auch gegen den Vorwurf zur Wehr setzt, der präventivmedizinischen Bewegung mangele es an einer „explicit basis in scientific theory“.151 MacKenzie, seit 14 Jahren im öffentlichen Gesundheitsdienst tätig, verwies auf die beeindruckenden Ergebnisse jener eingeleiteten „hygienic renascence "152 und demonstrierte, dass auch die präventivmedizinische Bewegung auf wissenschaftlichen, vor allem medizinisch-biologischen Theorien beruhte:

The hygienic renascence is the objective side of a movement whose ethical basis is the set effort after a richer, cleaner, intenser life in a highly organised society. The postulates of hygienics whose administrative form constitutes the public health service - are such as these: that society or the social group is essentially organic; that the social organism, being as yet but little integrated, is capable of rapid and easy modification - that is, of variations secured by selection; that disease is a name for certain mal-adaptations of the social organism or of its organic units; that diseases are thus, in greater or lesser degrees, preventable; that the prevention of diseases promotes social evolution; that, by the organisation of representative agencies - county councils, town councils, district councils, parish councils and the like - the processes of natural selection may be indefinitely aided by artificial selections; that thus, by continuous modification of the social organism, of its organic units and of the compound environment of both, it is possible to further the production of better citizens - more energetic, more alert, more versatile, more individuated. Provisionally, public health may be defined as the systematic application of scientific ideas to the extirpation of diseases, and thereby to the direct or indirect establishment of beneficial variations both in the social organism and in its organic units. In more concrete form, it is an organised effort of the collective social energy to heighten the physiological normal of civilised living. ${ }^{153}$

MacKenzie wollte nicht in Abrede stellen, dass Eugeniker und Präventivmediziner im Grunde das gleiche Ziel verfolgten, nämlich ,the production of better citizens". ${ }^{154}$ Nach seiner Auffassung gingen beide aber von grundverschiedenen Annahmen aus, denn die Präventivmediziner setzten auf das menschliche Anpassungsvermögen. Sie würden darauf beharren, „that the human organism is capable of greater things than on the average it has anywhere shown, and that its potentialities can be elicited by the systematic improvement of the environment. From the practical side, hygienics aims at,preparing a place'for the highest average of faculty to develop in. "155 Welche Fähigkeiten und Potentiale auch immer in der menschlichen Anlage schlummern mochten, ohne ein entsprechendes günstiges Umfeld, so befand MacKenzie, konnten diese nicht geweckt und entfaltet werden. Den Präventivmedizinern ginge es deshalb ganz utilitaristisch darum, die Umwelt

150 William Leslie MacKenzie in Sociological Papers (1904), S. 65-66; siehe auch ders., Health and Disease, London 1911, und MacKenzies Besprechung von William Healys The Individual Delinquent, in: Mind 28 (1919), S. 354-358.

151 MacKenzie in Sociological Papers 1 (1905), S. 66.

152 Ebd., S. 66.

153 Ebd., S. 66, Hervorhebungen S.F.

154 Ebd.

155 Ebd., Hervorhebung S.F. 
möglichst vieler Menschen so positiv zu verändern, dass vorhandenes Potential überhaupt die Chance bekam, sich entwickeln zu können.

Über das, was ,Anlage‘ generell beinhaltete, ließen sich kaum Aussagen machen. Deshalb warnten Präventivmediziner wie MacKenzie auch davor, angeborene und erworbene Eigenschaften miteinander zu verwechseln. Zum Tragen kamen in diesen Diskussionen die neuesten Erkenntnisse der Bakteriologie. Am Beispiel der Tuberkulose demonstrierte MacKenzie, dass erst die Entdeckung eines bakteriellen Erregers mit der alten Vorstellung aufgeräumt habe, Tuberkulose sei erblich bedingt und erkrankte Paare täten gut daran, keine Nachkommen zu zeugen. Auch müsse bedacht werden, „that the vast majority of diseases are due either to the attacks of infective or parasitic organisms or to the functional stress of environment, which for this purpose is better named ,nurture." 156 Eine von ihm durchgeführte Untersuchung an Schulkindern hatte MacKenzie davon überzeugt, dass ererbte Eigenschaften, gute wie schlechte, in jeder sozialen Klasse durch die Einwirkungen der Umwelt so verdeckt seien, dass nicht angegeben werden könne, was auf nature und was auf nurture zurückzuführen sei. ${ }^{157}$ Hier eröffne sich nun ein weites Feld der Forschung.

Die Haltung der meisten Präventivmediziner war damit klar umrissen: Solange die Vererbungsmechanismen nicht eindeutig geklärt waren, würden Ärzte darin fortfahren, dort einzugreifen, wo sie mit ihren Mitteln Erfolge erzielen konnten. Keiner dieser Mediziner zweifelte daran, dass es ernsthafte Erkrankungen gab, von denen mit gutem Recht angenommen werden musste, dass sie erblich bedingt waren. Aber diese Krankheiten waren weder das zentrale Problem des sozialen Elends der unteren Klassen im Allgemeinen noch von Kriminellen im Besonderen.

\section{The establishment of beneficial variation. Zur wissen- schaftlichen Kritik von innen}

Gerade weil es ein Anliegen der Eugenics Education Society war, ihr Programm wissenschaftlich zu legitimieren, warb sie besonders um die Mitgliedschaft von Wissenschaftlern. Junge, aufstrebende Biologen, Mediziner, Bio-Statistiker und Anthropologen wie Ronald A. Fisher, Alexander Carr-Saunders, Julian Huxley, John B.S. Haldane, George Pitt-Rivers und Marie Stopes konnten in der Anfangs-

156 Ebd., S. 67.

157 Ebd., Hervorhebung S.F.; um seinen Standpunkt zu unterstreichen und gleichzeitig ein neues Argument in die Debatte über die von Eugenikern mit Sorge betrachtete, angebliche differential birth rate einzuführen, verwies MacKenzie abschließend auf die Züchtungsexperimente seines Kollegen Dr. Noel Paton mit Meerschweinchen, die gezeigt hätten: „that starved mothers produce starved offspring and that well-fed mothers produce well-fed offspring. [...] the numbers of offspring were unaffected. "Statt eine abnehmende Reproduktionsfähigkeit in den höheren Klassen anzunehmen, müsse vielmehr angenommen werden, „that reproduction may be limited by a different ethic. The universal fall in the birth-rate has been too rapid to justify simpliciter the conclusion that biological capacity has altered." (Ebd. S. 67, Hervorhebung S.F.). 
phase ebenso gewonnen werden wie die Psychiater und Psychologen Alfred Tredgold, Cyril Burt, William McDougall und Havelock Ellis und der Ökonom John Mayard Keynes. ${ }^{158}$ Mit Blick auf die in der Gesellschaft vertretenen Mediziner muss allerdings differenziert werden: Mediziner stellten zwar einen Großteil des Vorstandes und des Beirates, unter den einfachen Mitgliedern waren sie aber eher spärlich vertreten. Bei den Beiratsmitgliedern handelte es sich zudem eher um Männer, die wie Havelock Ellis oder Caleb Saleeby, vor allem als Autoren wissenschaftlicher Bücher und als Journalisten tätig waren, und Forschung in populärer Form vermittelten. Die große Masse praktizierender Ärzte blieb der Eugenics Education Society fern, wie auch die British Medical Association ihr gegenüber feindselig eingestellt blieb und es zu verhindern wusste, dass Eugenik Teil des medizinischen Curriculums wurde. ${ }^{159}$

Die jungen, aufstrebenden Naturwissenschaftler erhofften sich durch ihre Mitgliedschaft bessere Karrierechancen, denn ihnen fehlte bis dahin, wie Dorothy Porter argumentiert, „direct access to such traditional channels of social influence as the media, politics, administration, or industrial relations". 160 Viele von ihnen brachten dezidierte Forschungsinteressen mit und hofften auf Synergieeffekte, die sich durch die Beschäftigung mit Eugenik für ihre eigenen Forschungsgebiete (z.B. Alkoholismus, Epilepsie) ergeben sollten. Die Eugenics Education Society finanzierte Projekte und eröffnete Publikationsmöglichkeiten. ${ }^{161}$ Gerade den Vertretern der jungen Disziplinen wie Demographie, Statistik, Psychologie und natürlich Genetik ${ }^{162}$ schien Eugenik als angewandte Wissenschaft eine erhöhte Nachfrage ihrer Expertise in naher Zukunft in Aussicht stellen zu können.

Doch Motive wie Profilierung und Statussicherung der eigenen Disziplin bedeuteten keineswegs, dass diese jungen Wissenschaftler das sozialpolitische Programm der Eugenics Education Society vorbehaltlos oder unkritisch unterstützten. Als Beispiel dafür kann hier der Vortrag des Gefängnisarztes William C. Sullivan ${ }^{163}$ über Eugenics and Crime angeführt werden, der 1909 in der ersten Ausgabe der Eugenics Review erschien. Sullivan arbeitete zu diesem Zeitpunkt als Medical Officer im Holloway Prison, kletterte aber bald die Karriereleiter im öffentlichen Dienst nach oben und wurde später Leiter des Broadmoor Criminal Lunatic Asylum und damit Nachfolger von David Nicolson. ${ }^{164}$ Zusammen mit den Ärzten Sir James Crichton-Browne, Archdall Reid und Frederick Mott war

158 Allerdings ließ Keynes' Galton Lecture 1937 überhaupt keinen Bezug zur Eugenik erkennen, was besonders Leonard Darwin verstörte, siehe RICHARD SOLOWAY, From Mainline to Reform Eugenics - Leonard Darwin and C. P. Blacker, in: Galton Institute (Hrsg.), Essays, S. 69.

159 Mazumdar, Eugenics, S. 9; Radzinowicz und Hood, The Emergence of Penal Policy, S. 324.

160 Porter, Enemies of the Race, S. 164; Porter übernimmt hier ein Argument von Searle, Eugenics and Class, S. 235-238.

161 Searle, Eugenics and Class, S. 222.

162 Der Biologe William Bateson prägte den Begriff genetics in einer Korrespondenz des Jahres 1905, seit 1908 war er der erste Professor of Genetics in Cambridge, siehe dazu ausführlich Kap. 5.3.

163 Zu Sullivan siehe auch DavIE, Tracing the Criminal, S. 257f.

164 Zu Nicolson ausführlich Kap. 3 dieser Arbeit. 
Sullivan nicht nur Mitglied der EES, sondern gehörte auch der 1884 gegründeten Society for the Study of Inebriety an, einer fast ausschließlich aus Medizinern zusammengesetztem Vereinigung, die Fragen von Alkoholismus unter sozialen, erblichen und juristischen Aspekten untersuchte. ${ }^{165}$ Von Anfang an verlor Sullivan in seinem Vortrag den juristischen und sozialpolitischen Bezug zur Kriminalität nicht aus den Augen. Gegen die im Umlauf befindlichen eugenischen Definitionen betonte er die normativen Kriterien, die die Kategorie des Straftäters bestimmten. Es sei unmöglich, befand Sullivan, den Begriff des Kriminellen durch rein biologische Begriffe $\mathrm{zu}$ fassen. ${ }^{166}$ Was als kriminelle Handlung angesehen werde, sei die Verletzung von Normen, die ihrerseits kulturell vermittelt und damit auch veränderbar seien.

Wie Gefängnisärzte und -psychiater vor ihm wandte sich Sullivan vor allem gegen die von vielen Eugenikern unkritisch angeführte Gleichsetzung von Kriminellen und Geisteskranken. Gerade der Vergleich mit Geisteskranken zeige doch, wie unterschiedlich sich beide Erscheinungsformen ausnähmen:

In insanity the sexual incidence is sensibly equal; in crime, on the contrary, there is a predominance of males, corresponding to the greater part taken by men in social and industrial activities and similar in amount to the predominance of males in suicide and in alcoholism. Again, the statistical movement of insanity shows relatively little trace of the influence of those industrial changes which so potently affect the volume and character of crime. ${ }^{167}$

Während bei Männern und Frauen in einem ähnlichen Verhältnis Geisteskrankheiten auftraten, zeige sich in kriminellen Handlungen eine ausgeprägte männliche Dominanz - das hatten ja die Statistiker im 19. Jahrhundert schon herausgestellt. Ökonomische Schwankungen und ihre Auswirkungen auf den Verlauf von Kriminalitätskurven waren für Sullivan ein Indiz für den enormen Einfluss äußerer Faktoren auf die Kriminalitätsgenese, vor allem auf die primär in diese Prozesse eingebundene männliche Bevölkerung: „criminals, unlike lunatics but like suicides and alcoholics, are made rather than born [...] in their genesis environment plays a larger part than innate predispositions. "168 Sullivan hielt deshalb das Verhältnis von Kriminalität und Eugenik für „somewhat restricted. “169

Als Arzt stellte auch Sullivan nicht in Abrede, dass es gefährliche Delinquenten gab, die geisteskrank oder -gestört waren. Aber auch bei ihnen könne man nicht davon ausgehen, dass sie durch Vererbung in ihren Handlungen determiniert seien. Was diese pathologischen Fälle auszeichne, sei nicht geerbte Kriminalität, sondern durch Krankheit bedingte geistige Unfähigkeiten und Beschränkungen. Das

165 William C. Sullivan, Alcoholism, London 1906; zu den Doppel- und Mehrfachmitgliedschaften siehe MazumDar, Eugenics, S.30f.; zur Diskussion über Alkoholismus und seine Ursachen zwischen EES und Eugenics Laboratory und zur anfänglichen Annäherung der EES an die Society for the Study of Inebriety siehe GiLlham, Francis Galton, S. 336-340; Crichton-Browne war zeitgleich erster Präsident der EES und Vize-Präsident der Society for the Study of Inebriety.

166 William C. Sullivan, Eugenics and Crime, in: Eugenics Review 1 (1909), S. 112-120.

167 Sullivan, Eugenics, S. 115.

168 Beide Zitate: ebd., S. 116.

169 Beide Zitate: ebd. 
Fehlen sozialer Kompetenz, um soziales Verhalten zu erlernen, existiere, so argumentierte Sullivan, nicht als isolierte Bedingung, sondern sei bestenfalls eine Seite der „general debility of mind“"“170 Hier habe man es primär auch nicht mehr mit Kriminellen, sondern mit kranken Patienten zu tun, die sozusagen eine „artificial group inside the great pathological class of the feeble-minded“ bildeten. ${ }^{171}$ Während für Sullivan vor allem Männer „of average stock“ ${ }^{172}$ unter dem Einfluss eines bestimmten Milieus kriminell wurden - sie brauchten den Eugeniker ohnehin nicht zu interessieren -, sei die kleine Gruppe pathologischer Verbrecher ausnahmslos ein Fall für Ärzte und Psychiater, also für Leute wie Sullivan selbst und seine Kollegen. Die von Staatswegen in solchen Fällen angeordnete Unterbringung in speziellen Institutionen sorge ohnehin für eine negative Eugenik.

Das letzte von Sullivan in seinem Vortrag angeführte Argument war vielleicht das wichtigste: Er wies darauf hin, dass es neben der großen Gruppe gewöhnlicher und der kleinen Gruppe kranker Krimineller auch noch eine dritte Gruppe gebe: den brillanten, einfallsreichen Berufsverbrecher „of good stock“. Dessen Talent und Begabung - ,skilled criminals are those in which energy and initiative are most abundant" 173 - auszurotten, könne für eine Gesellschaft überhaupt nicht wünschenswert erscheinen, sie müsse, im Gegenteil, den Versuch unternehmen, durch eine entsprechende Umerziehung im Gefängnis die Talente dieses Straftäters für die Gemeinschaft fruchtbar zu machen.

Mit diesem Argument befand sich Sullivan in guter Gesellschaft einiger weiterer wissenschaftlicher Mitglieder der EES. Schon 1904 hatte H.G. Wells, Naturwissenschaftler, Schriftsteller und Mitbegründer des Royal College of Science, gegen Galton eingewendet, er sehe sich nicht dazu veranlasst, Kriminelle generell als Träger negativer Qualitäten zu betrachten:

I am not even satisfied by the suggestion Dr. Galton seems to make that criminals should not breed. I am inclined to believe that a large proportion of our present-day criminals are the brightest and boldest members of families living under impossible conditions, and that in many desirable qualities the average criminal is above the average of the law-abiding poor, and probably of the average respectable person. Many eminent criminals appear to me to be persons superior in many respects, in intelligence, initiative, originality, to the average judge. ${ }^{174}$

Wells war es auch, der auf das soziale Umfeld im Sinne einer vorteilhaften Vernetzung der Familie aufmerksam machte, die beim beruflichen Fortkommen wichtiger schien als „any distinctive family gift. “175 Auch der einflussreiche Verhaltenspsychologe William McDougall hatte die Beurteilung des Sozialverhaltens im Blick, als er erklärte, Kriminelle seien überhaupt keine Degenerierten, „but merely non-conformists“. Auch McDougall warnte: „[T]o sterilise in any way our crimi-

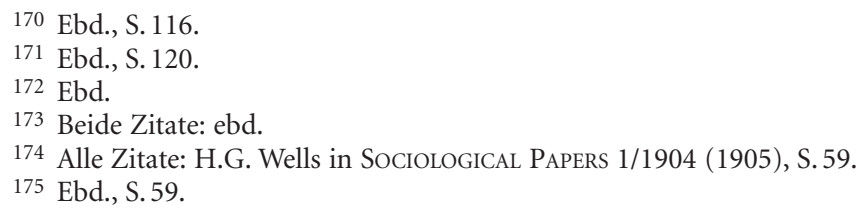


nal stocks would be to eliminate our most variable stocks“, schließlich sei „variability [...] the prime condition of all evolution"176.

Die klare Grenzziehung zwischen Kriminalität und Krankheit und der Hinweis auf den begabten, talentierten Kriminellen durch drei, der eugenischen Sache durchaus aufgeschlossene Autoren und Wissenschaftler, waren wichtige Stellungnahmen: Hier wurde das biologische Konzept der Variation (variability) aus der Evolutionstheorie gegen die von den Eugenikern versuchte Hierarchisierung menschlicher Eigenschaften und Fähigkeiten und gegen eine unterschiedliche Wertung der verschiedenen gesellschaftlichen Klassen ins Feld geführt. Gerade die Vorstellung von Variation als eines für das Überleben und Fortkommen einer Population wichtigen evolutionären Merkmals (die Toleranz gegenüber einer sich ändernden Umwelt wurde erhöht) war anschlussfähig an das Bestreben der Präventivmediziner, durch „the establishment of beneficial variations"177 bessere Bürger hervorzubringen. Die Betonung von Abweichung, Abwechslung und Vielfältigkeit innerhalb einer Population konnte politisch als Bekenntnis zur Pluralität der Gesellschaft genutzt werden.

Unter den wissenschaftlichen Mitgliedern der Eugenics Education Society wurde nicht nur die Frage gestellt, wer die Standards der besonders förderungswürdigen menschlichen Eigenschaften festlegen sollte, sondern auch, wie eine solche Liste überhaupt zustande kommen sollte. Ironischer Weise wurde diese Überlegung recht früh von Max Nordau formuliert, dessen zentrales, Cesare Lombroso gewidmetes Werk Entartung die Debatten über Degeneration maßgeblich beeinflusst hatte. ${ }^{178}$ In seinem schriftlichen Kommentar zu Galtons Vortrag von 1904 heißt es:

It is clear that we cannot apply the principles of artificial breeding to man. There is no recognised standard of physical and intellectual perfection. Do you want inches? In that case, you would have to exclude Frederick the Great and Napoleon I, who were undersized; Thiers, who was almost a dwarf; and the Japanese as a nation, as they are considerably below the average of some European races. ${ }^{179}$

Differenzierter als Nordau formulierte 1913 der Biologe, Soziologe und Demograph Alexander Carr-Saunders als Mitglied der EES erneut diese Einwände. ${ }^{180}$

176 William McDougall, A Practicable Eugenic Suggestion, in: Sociological Papers 2/1905 (1906), S. 57; nach HEARNSHAw, Short History of British Psychology, S. 186, war McDougall der einflussreichste Sozialpsychologe in England vor dem Zweiten Weltkrieg, siehe dazu auch Kap. 7.2.

177 MacKenzie in Sociological Papers 1/1904 (1905), S. 66.

178 Vgl. Max Simon Nordau, Entartung, Berlin 1892; die deutsche Ausgabe widmete Nordau Cesare Lombroso, die englische Ausgabe Degeneration erschien 1895; im gleichen Jahr erschien eine anonyme Kritik an Nordaus Werk mit dem Titel Regeneration: a Reply; zu Nordau siehe PICK, Degeneration, S. 24-26; Nordau befasste sich vor allem mit dem Phänomen Hysterie. Er verstand unter einem entarteten Künstler (z. B. Richard Wagner) nicht einen biologisch degenerierten, sondern einen irrationalen Künstler, der sich in Traumwelten verlor und sich seinen innerlichen Phantasien überließ; in der Musik sah er dann Chaos an die Stelle von Ordnung und Rhythmus treten.

179 Kommentar von Max Nordau in Sociological Papers 1/1904 (1905), S. 30-33, hier S. 31.

180 Vgl. z. B. Alexander Carr-Saunders, The Population Problem, London 1922; ders. World Population, Oxford 1936; 1923 wurde Carr-Saunders der erste Inhaber des Charles Booth Chair of Social Science in Liverpool, 1926 war er Vorsitzender des von der EES ins Leben 
Carr-Saunders setzte sich zunächst mit dem Stand der biologischen Erbforschung auseinander und kam zu dem Ergebnis, dass die Wissenschaft noch gar nicht in der Lage sei, auch nur annähernd den komplexen Vorgang der Weitergabe menschlicher Eigenschaften - darunter die für Eugeniker besonders wichtigen Eigenschaften der Leistungsfähigkeit und Intelligenz - zu erklären, geschweige denn auf dieser Grundlage eine bestimmte Politik zu legitimieren. ${ }^{181}$ Aber nicht nur die biologischen Voraussetzungen seien zu wenig erforscht, auch der Einfluss der Umwelt sei noch nicht ausreichend aufgeschlüsselt. ${ }^{182}$

Im politisch brisanteren Teil seines Aufsatzes unterzog Carr-Saunders die von den Eugenikern immer wieder bemühten menschlichen Qualitäten, die es durch positive Eugenik zu fördern gelte, einer genaueren Prüfung. ${ }^{183}$ Eugeniker hatten sich mit einer genaueren Bestimmung dieser Eigenschaften stets bedeckt gehalten. In seinem Vortrag von 1904 hatte Galton nur leichthin behauptet: „A considerable list of qualities can be easily compiled [...] It would include health, energy, ability, manliness and courteous disposition." 184 Carr-Saunders störte besonders, dass die Eugeniker von einer „superiority of inherited characters in the so-called upper classes" so selbstverständlich ausgingen. Sie wäre aber ebenso wenig bewiesen wie die Annahme, dass die unteren Klassen den oberen gegenüber „inferior“185 seien. Selbst wenn es möglich wäre, sich auf eine Liste der für eine Gesellschaft wichtigsten menschlichen Eigenschaften zu einigen, so müssten diese als in allen Klassen vorhanden angenommen werden. Generell aber stellte Carr-Saunders den Sinn einer gezielten Förderung bestimmter menschlicher Eigenschaften überhaupt in Frage. Wer wolle entscheiden, welche Eigenschaften für eine sich stets im Wandel befindliche Gesellschaft die wichtigsten seien? Wäre die Herstellung bestimmter Typen für eine Gesellschaft überhaupt erstrebenswert? Läge in einer solchen Einseitigkeit nicht geradezu eine Gefahr? Auch Carr-Saunders operierte in seinen Ausführungen mit der Vorstellung von einer notwendigen und sinnvollen Variation menschlicher Fähigkeiten und Potenziale in einer pluralistischen Gesellschaft, die nicht nur deren Flexibilität garantieren, sondern auch ihre Anpassungsfähigkeit steigern konnte.

Durch seine Einwände wollte Carr-Saunders nicht die Wichtigkeit eugenischer Forschung schmälern, im Gegenteil, die vielen offenen Fragen verlangten nach einer noch viel intensiveren Forschung über den Einfluss von Anlage und Umwelt

gerufenen Population Investigation Committee, in den 1930er Jahren wurde er Direktor der London School of Economics and Social Sciences und von 1944-1949 hatte er den Vorsitz des Statistics Committee of the Royal Commission on Population inne, siehe Mazumdar, Eugenics, S. 124.

181 Alexander M. Carr-Saunders, A Criticism of Eugenics, in: Eugenics Review 5 (1913), S. 214-233; siehe auch Carr-Saunders Besprechung von Charles Gorings The English Convict in: ders., Eugenics, London 1926, Kap. 7: The Distribution of Inherited Qualities, darin Abschnitt über „Crime“, S. 142-153.

182 CARr-Saunders, Criticism, S. 220.

183 Ebd., S. 221.

184 GalTon, Eugenics: Its Definition, S. 46.

185 CARr-Saunders, Criticism, S. $226 \mathrm{f}$. 
auf das menschliche Verhalten. Dagegen glaubte er die Instrumentalisierung eines nicht abgesicherten Wissens für ein sozialpolitisches Programm nicht rechtfertigen zu können: „justification is at present lacking. “186 Nicht zuletzt die Wissenschaft selbst würde dadurch korrumpiert werden und an Glaubwürdigkeit einbüßen. Nach dem Ende der Präsidentschaft Leonard Darwins 1929 gehörte CarrSaunders zur Gruppe jener Reformeugeniker um Charles P. Blacker, die gegen den Widerstand zahlreicher Mitglieder die EES langsam auf den Kurs einer learned society brachte, die sich nun stärker auf die Vermittlung und Förderung ihrer Wissenschaft, besonders bevölkerungsdemographischer Untersuchungen und genetischer Forschung, konzentrierte. ${ }^{187}$

\section{„Our Critic Criticized“. Die Antwort der Eugeniker}

Wie reagierten Eugeniker wie Leonard Darwin auf die zum Teil von den eigenen Mitgliedern vorgetragene Kritik? Darwin selbst ließ sich auf eine Diskussion über die wissenschaftlichen Grundlagen der Eugenik nicht ein. In der Vorstellung gefangen, dass die Öffentlichkeit klare Vorschläge zur Beseitigung sozialer Probleme erwarten dürfe - er verstand seine Arbeit ja als „service to mankind“ 188 -, nahm er die Vereinfachung des vermittelten Wissens mit dem Hinweis auf die Zielsetzung des Programms der der Eugenics Education Society bewusst in Kauf. Man betreibe das Studium evolutionärer Faktoren, die den Fortschritt der Rasse verantwortlich gemacht werden könnten, letztlich nur deswegen, so argumentierte Darwin, weil man das auf diese Weise erworbene Wissen für den Fortschritt der Menschheit einsetzen wolle. An den allgemeinen Prinzipien der Evolution bestand seiner Auffassung nach kein Zweifel. Ihre allgemeine Anerkennung hielt er für ausgemacht. Natürlich gab Darwin zu, dass noch nicht alle Fragen in Bezug auf die Evolution geklärt waren, aus seiner Sicht erlaubte die Dringlichkeit der Reformen aber keinen Aufschub: „It is therefore obvious that we cannot wait until all doubts are removed, as that would probably mean postponing all reform for ever."189

Ähnliche Argumente wurden auch von anderen Eugenikern angeführt. Für Ferdinand Schiller, ebenfalls kein Naturwissenschaftler, sondern Philosophieprofessor in Oxford, stand außer Frage, dass der praktisch orientierte Eugeniker nicht darauf warten könne, bis sein Wissen den - ohnehin nur illusionären - Stand der Vollkommenheit erreicht hätte. Man brauche doch nur ein relativ bescheidenes $\mathrm{Maß}$ an Wissen, um in die Lage versetzt zu werden, das diagnostizierte soziale Übel effizient anzugehen. Kurzum: „True, I know little enough, but I know enough

186 Ebd., S. 233.

187 Zur weiteren Geschichte der Eugenics Society besonders nach dem Ersten Weltkrieg sehr aufschlussreich SolOWAY, From Mainline to Reform Eugenics, S. 52-80; MACNICOL, Eugenics; zur allgemeinen Entwicklung und der abnehmenden öffentlichen Präsenz der EES siehe auch FreitaG, We Cannot Wait, S. 310-312.

188 Vgl. Darwin, Eugenics Reform, S. 1 (Widmung für seinen Vater).

189 LeOnard Darwin, Our Critic Criticized, in: Eugenics Review 5 (1913), S.316-325, hier S. 317 . 
to start on; moreover, it is only by experimenting and taking certain risks that I can ever hope to learn what as yet I do not know. "190 So wie Leonard Darwin die Prinzipien der Evolution zum Ausgangspunkt seiner Argumentation machte, so setzte Schiller bei der Existenz vererbbarer Defekte und der offensichtlichen Möglichkeit ihres ,Herauszüchtens' an: „It is enough that the existence of hereditary defects should be admitted, and that it is possible to get rid of them. " ${ }^{191}$ Der Eugeniker brauche keine endgültigen Erkentnnisse über das genaue Verhältnis, „in which ,nature' and ,nurture ' may be held to contribute to a man's total character." ${ }^{192}$ Die Details der wissenschaftlichen Kontroversen seien, so Schiller, für seine Arbeit nicht relevant.

Mit dieser Haltung erklärten sich Eugeniker wie Darwin und Schiller in gewisser Weise ein Stück weit von der Forschung unabhängig: „In like manner the biological disputes between the Lamarckians, Darwinians, Mendelians, and Biometricians, and the unsolved problem which they rage, are, for practical purposes, largely irrelevant. "193 Genau darin täuschten sie sich aber. Gerade die Debatten zwischen den Biometrikern und den Anhängern Mendels sollten ganz entscheidenden Einfluss auf den Fortbestand und das Selbstverständnis der EES haben und nicht zuletzt auch in den zeitgenössischen Debatten über Kriminalitätsgenese und Verbrecherkonstitution Spuren hinterlassen. ${ }^{194}$ Allgemeiner Widerstand ließ sich aber an vielen Orten antreffen. So brachte 1913 Abgeordnete Josiah Wedgewood in den parlamentarischen Diskussionen über den Mental Deficiency Act ${ }^{195}$ eine Klausel zu Fall, die das Verbot festschrieb, eine/n mental defective heiraten zu können. Wedgewood warnte davor, nichts in ein Gesetz umzuwandeln „for the sake of a scientific creed which in ten years may be discredited“. ${ }^{196}$ Generell verurteilte er in diesem Zusammenhang „the most gross materialism that has ever been imported into human society“. 197

\subsection{Bilanz und Ausblick: Regeneration versus Degeneration}

Was bedeutete die Debatte zwischen Allgemeinmedizinern und Eugenikern für den Diskurs über Kriminalität und Verbrecherkonstitution? Zunächst einmal haben die wissenschaftlichen Einwände gegen die nicht gesicherten Erkenntnisse zur Vererbung menschlicher Eigenschaften dazu beigetragen, dass sich in Bezug

190 Ferdinand C.S. Schiller, Our Critic Criticized, in: Eugenics Review 5 (1913), S.325-333, hier S. 326f.

191 Ebd., S. 327.

192 Ebd.

193 Ebd., S. 328.

$194 \mathrm{Zu}$ dieser Auseinandersetzung siehe Kap. 5.3.

195 Zum Mental Deficiency Act siehe Kap. 3, Anm. 121.

196 Josiah Wedgewood zit. nach RadzINowicz und Hood, The Emergence of Penal Policy, S. 337f.

197 RadzinOwicz und Hood, The Emergence of Penal Policy, S. 336. 
auf Kriminalität und Eugenik der Ton der Eugenics Education Society sehr verhalten ausnahm. Aufgrund der großen Wissenslücken hielten sich nicht nur Präventivmediziner, sondern auch einige der wissenschaftlichen Mitglieder der EES mit Aussagen über eine angebliche kriminelle Disposition zurück. Ihre Einwände bremsten, sogar langfristig, die voreilige Forderung einiger britischer Eugeniker nach Sterilisation und Separierung von Straftätern zur Unterbindung ihrer Reproduktionsfähigkeit.

Kriminalität, dies war vor allem im Bewusstsein der in staatlichen Diensten stehenden Mediziner verankert, war immer auch etwas entschieden Anderes als Krankheit. Mochte bei Geisteskranken, deren Existenz kein Mediziner in Frage stellte, auch über die dauerhafte Unterbringung in Heimen oder über andere Formen der sozialen Kontrolle öffentlich nachgedacht werden, die prinzipielle Frage blieb, ob Geisteskranke mit Kriminellen auf eine Stufe gestellt werden konnten. Viele Ärzte verneinten dies und sorgten durch ihre Einstellung für die Aufrechterhaltung des Unterschieds zwischen Krankheit und Kriminalität bzw. zwischen mentalen Störungen und kriminellem Verhalten. Im Einklang mit der Auffassung von Regierung und Rechtsprechung wurden Straftäter, mit Ausnahme einer kleinen Minderheit geistig kranker Personen, als für ihre Handlung verantwortliche und schuldfähige Subjekte angesehen. Sie sollten durch staatliche Sanktionen nicht entmündigt, sondern als Bürger mit Rechten und Pflichten weiterhin ,ernst genommen werden, und das hieß auch, Ihrer Strafe zugeführt werden.

Der Versuch, eugenische Prinzipien auf die kleine Gruppe der feeble-minded anzuwenden bzw. die Mehrheit der Straftäter dieser feeble-minded-Gruppe zuzurechnen und durch deterministische Annahmen schlechte Sozialprognosen für Straftäter auszusprechen, provozierte den Widerstand einer Reihe professioneller und privater Bewegungen, die ganz entschieden auf die Möglichkeiten positiven Eingreifens setzten. „Galton's plea for a science of eugenics“, so hat es einer der schärfsten Kritiker der Eugenik, der Sozialbiologe und Mathematiker Lancelot Hogben in seinem 1938 erschienenen Buch Science for the Citizen in der Rückschau formuliert, „became identified with a system of ingenious excuses for combating the amelioration of working-class conditions." 198

Leonard Darwin hat 1914 den Widerstand dieser Environmentalisten, Philanthropen und Sozialreformer deutlich gesehen und ihren Einfluss noch mehr gefürchtet als die Kritik der Mediziner:

In the case of those who have for long been devoting their best efforts to the study of questions immediately affecting human environment - in other words in the case of nearly all philanthropists - the diversion of their attention to the question connected with heredity seems to require a severe mental wrench, an effort which many of them appear to be wholly incapable of making. In short, many persons of the highest character have not yet learned to think eugenically. As to those whose thoughts are exclusively devoted to the betterment of their own personal surroundings, a change in their mental attitude is almost past praying for. ${ }^{199}$

198 Lancelot Hogben, Science for the Citizen. A Self-Educator based on the Social Background of Scientific Discovery, London 1938, S. 1054.

199 Darwin, Habitual Criminal, 204, Hervorhebung S.F. 
Der Präsident der Eugenics Education Society, der selbst eine tiefer gehende Beschäftigung mit den wissenschaftlichen Grundlagen der Eugenik vermied, beschwor mit diesem Verweis das Bild einer elementaren Auseinandersetzung zwischen aufgeklärten Eugenikern und immer noch verblendeten, unwissenschaftlichen, sentimentalen Philanthropen.

Diese Bild entsprach jedoch nicht der Wirklichkeit. Zur Kenntnis genommen wurde von den Philanthropen nämlich auch der unsentimentale, ebenfalls mit wissenschaftlichem Anspruch auftretende Diskurs der mehrheitlich in staatlichen Diensten angestellten Präventivmediziner. Während Eugenik bei der Hervorbringung ,besserer' Bürger auf Selektion und Ausgrenzung setzte, baute die Präventivmedizin auf Integration auch der schwächeren Teile der Bevölkerung und auf die Anhebung ihrer unmittelbaren Lebensumstände. Dem Phänomen Degeneration wurde das Modell Regeneration entgegengesetzt. „Medical men“, so der Mediziner Archdall Reid in seiner Antwort auf Galtons Vortrag von 1904, „have done a good deal for the improvement of [...] the individual by improving sanitation. They have attempted nothing towards [...] the improvement of the inborn qualities of the race. Nor will they attempt anything until they have acquired a precise knowledge of heredity from biologists. "200 Aus Sicht der Ärzte waren die Biologen und Eugeniker in der Bringschuld. ${ }^{201}$ Solange die Weitergabe menschlicher Eigenschaften nicht geklärt sei, würden Ärzte darin fortfahren, mit den ihnen zur Verfügung stehenden Mittel an der „production of better citizens“202 weiterzuarbeiten. Dabei würden sie - zugegebenermaßen - zusammen mit den Sozialreformern am scheinbaren Paradox der Wohlfahrt weiterwirken, indem sie auch jenen Unterstützung zukommen ließen, die in einem natürlichen Selektionsprozess offensichtlich zum Untergehen bestimmt seien.

Für dieses sozialpolitische Programm der Regeneration konnten sich Präventivmediziner ebenfalls auf Konzepte der Evolutionsbiologie berufen: auf die enorme Anpassungsfähigkeit menschlicher Wesen an eine sich ändernde Umwelt sowie auf die Theorie des evolutionären Vorteils von Variation, die die einseitige Förderung von Eigenschaften als evolutionären Nachteil eher verbot. Welche Anlagen in welchen Schichten zu finden waren und als besonders vorteilhaft eingestuft werden mussten, ließ sich schon deshalb nicht beantworten, weil die unteren Schichten bislang wenig Möglichkeiten gehabt hätten, die ihren zur Entfaltung zu bringen. Auch die Vernachlässigung des Faktors Umwelt, die nach Ansicht der Mediziner für die Entfaltung von Anlagen als zentral betrachtet werden müsse, diskreditierte die Eugeniker in den Augen vieler Mediziner. Leonard Darwin selbst wusste um das damit verbundene Methodenproblem. Es handelte sich um eine

200 ARCHDALL ReID, Schriftlicher Kommentar zu Galtons Vortrag von 1904, in: Sociological Papers 1/1904 (1905), S. 72.

201 Siehe zur Ablehnung des eugenischen Programms durch Gefängnisärzte und -psychiater den Leitartikel von James Devon, The Eugenist and the Rest of Us, in: The British Medical Journal 2 (1912), S. 1641.

202 W. Leslie MacKenzie, Schriftlicher Kommentar zu Galtons Vortrag von 1904, in: Sociological Papers 1/1904 (1905), S. 66. 
Schwachstelle der eugenischen Stammbaumanalyse: „The collection of pedigrees of criminal and non-criminal families with proper care is of great value; but in drawing conclusions from them we are always brought face to face with the difficulty of eliminating the element of environment." 203

Welchen konkreten Einfluss hatten dagegen Galtons Intelligenzforschungen auf die Kriminalitätsdebatten? Ohne Zweifel leisteten sie einer Argumentation Vorschub, die sich auf die Konstitution des Straftäters konzentrierte. Allgemein verfestigte sich vor allem die Vorstellung, dass Intelligenz erblich sei. Wenn angenommen wurde, dass viele Straftäter zur Gruppe der feeble- oder weak-minded gehörten, und Kriminalität ihre Ursache in dieser geistigen Unterlegenheit hatte, dann ließ sich indirekt von einer erblichen kriminellen Disposition sprechen. Für diese Annahme bedurfte es keiner besonderen Rezeption der Forschungen von Cesare Lombroso, solche Vorstellungen entwickelten sich in England ganz eigenständig und unabhängig von den Forschungen des italienischen Arztes.

Was Galtons Entwicklung technischer Verfahren zur Identifizierung von Kriminellen betraf, so stellten die Ergebnisse der composite photography bereits früh das in L'uomo delinquente entwickelte Modell eines atavistischen Kriminellen, der sich an äußeren Merkmalen erkennen ließ, in Frage. Der Nachweis distinkter äuBerer Merkmale, die Straftäter von gesetzestreuen Bürgern unterschied, gelang nicht. Galton selbst glaubte, seine Annahmen aber trotzdem nicht revidieren zu müssen. In dem Maße, wie elementare äußere Unterscheidungskriterien zwischen Straftäter und gesetzestreuem Bürger verschwanden, wurde am Nachweis einer distinkten, mit geistigen Kapazitäten verbundenen inneren Disposition gearbeitet, die beide unterscheiden sollte. Für den Nachweis dieser inneren Disposition zur Geistesschwäche beim Straftäter bedurfte es aber anspruchsvollerer und subtilerer Methoden als der Stammbaumanalyse. Galtons zweite wissenschaftliche Errungenschaft, die biometrische Statistik, die über die Errechnung von Korrelationskoeffizienten äußere Faktoren zu inneren in Beziehung setzen konnte, schien dafür ein vielversprechender Weg.

203 DARWIN, Habitual Criminal, S. 208; persönlich hielt Leonard Darwin freilich „the benefits from changes in the environment" für "strictly limited“, siehe seine Besprechung von Alexander Patersons Across the Bridges, in: Eugenics Review 4 (1912), S. 210. 